\title{
Numerical differentiation for non-trivial consistent tangent matrices: an application to the MRS-Lade model ${ }^{\star}$
}

\author{
Agustí PÉREZ-FOGUET, Antonio RODRÍGUEZ-FERRAN \\ and Antonio HUERTA ${ }^{1}$ \\ Departament de Matemàtica Aplicada III, E.T.S. de Ingenieros de Caminos \\ Edifici C2, Campus Nord, Universitat Politècnica de Catalunya \\ E-08034 Barcelona, Spain. web: www.upc.es \ma3\lacan.html
}

\begin{abstract}
In Reference [1] the authors have shown that numerical differentiation is a competitive alternative to analytical derivatives for the computation of consistent tangent matrices. Relatively simple models were treated in that reference. The approach is extended here to a complex model: the MRS-Lade model $[2,3]$. This plastic model has a cone-cap yield surface and exhibits strong coupling between the flow vector and the hardening moduli. Because of this, derivating these quantities with respect to stresses and internal variables - the crucial step in obtaining consistent tangent matrices - is rather involved. Numerical differentiation is used here to approximate these derivatives. The approximated derivatives are then used 1) to compute consistent tangent matrices (global problem) and 2) to integrate the constitutive equation at each Gauss point (local problem) with the Newton-Raphson method. The choice of the stepsize (i.e. the perturbation in the approximation schemes), based on the concept of relative stepsize, poses no difficulties. In contrast to previous approaches for the MRS-Lade model, quadratic convergence is achieved, for both the local and the global problems. The computational efficiency (CPU time) and robustness of the proposed approach is illustrated by means of several numerical examples, where the major relevant topics are discussed in detail.
\end{abstract}

Key words: consistent tangent matrices; numerical differentiation; MRS-Lade model; quadratic convergence; computational plasticity; full Newton-Raphson method

\footnotetext{
ऋ Partially supported by CICYT, Spain. Grant contract: TAP98-0421

1 Corresponding author. e-mail: huerta@lacan.upc.es
} 


\section{Introduction}

Consistent or algorithmic (as opposed to continuum) tangent matrices are a key ingredient in computational plasticity [4]. They are needed to solve the so-called global problem (i.e. the elastoplastic boundary value problem) with quadratic convergence, via a full Newton-Raphson linearization.

The most involved step in obtaining consistent tangent matrices is computing the derivatives of the flow vector and the hardening moduli with respect to the stresses and the internal variables [1]. These derivatives are also required to achieve quadratic convergence with the full Newton-Raphson method for the local problem (i.e. the integration of the elastoplastic constitutive relation at the Gauss-point level).

In complex material models, these derivatives are difficult to obtain analyti-
cally, because there is a high coupling between stresses and internal variables. This is the situation, for instance, for the MRS-Lade model [2,3]. This model is used to describe the behaviour of granular materials, such as sand, under both low and high confinement stresses [5]. It features a yield surface consisting of a cone and a cap, hardening and softening variables based on dissipated

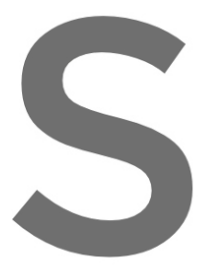
plastic work and a non-associated flow
region.
Because of the highly coupled nature
required derivatives are readily availabl
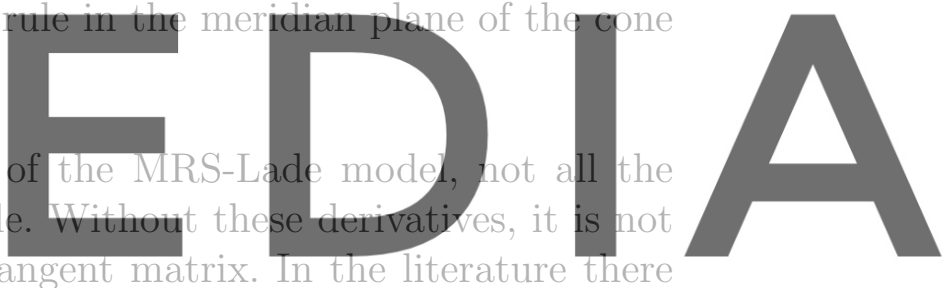
possible to compute a fuil consistent tangent matrix. In the literature ther Register for free at https/fwww. scipedia.com to download the version without the watermark involved to obtain than the derivatives of the stresses): 1) a tangent approach for the stresses and a direct substitution of the internal variable equations $[3,6]$ and 2) a two-level technique with a tangent approach for the stress invariants and a Picard iteration with an adaptive order inverse interpolation for the internal variables [7]. However, in both cases quadratic convergence is never achieved, because these approaches are not based on a consistent linearization of all equations with respect to all unknowns.

In the context of computational plasticity, Pérez-Foguet et al. [1] have shown that numerical differentiation is a simple and competitive alternative to classical analytical derivatives, provided that adequate schemes and stepsizes are chosen. It maintains the characteristic quadratic convergence of the NewtonRaphson method, both for the local and the global problems. In Reference [1], the results obtained with numerical differentiation are compared with those obtained with the analytical derivatives for two material models with analytical derivatives available: Von Mises and Rounded Hyperbolic Mohr-Coulomb. 
The main conclusion in [1] is that numerical differentiation is an efficient and robust strategy, which maintains quadratic convergence and has a very marginal CPU time overhead (with respect to analytical derivatives).

In this paper numerical differentiation is applied to compute the derivatives of a quite more complex model: MRS-Lade. In contrast to Reference [1], this model does not have all the analytical derivatives of the flow vector and the hardening moduli available. However, the same conclusions of Reference [1] are reached: robustness and efficiency.

Thus, the improvement of numerical differentiation over other techniques for the MRS-Lade model [6,7] is more clear than for simpler material models: it is the first time that quadratic convergence results are presented for the global and the local problems for this model. As a final introductory remark, it is worth noting that the MRS-Lade model is used here for illustrative purposes. The same approach can be used to compute consistent tangent matrices for other complex material models.

An outline of this paper follows. The problem is stated in Section 2. After some preliminaries on small-strain elastoplasticity, the MRS-Lade model is briefly reviewed. The proposed approach, based on numerical differentiation,
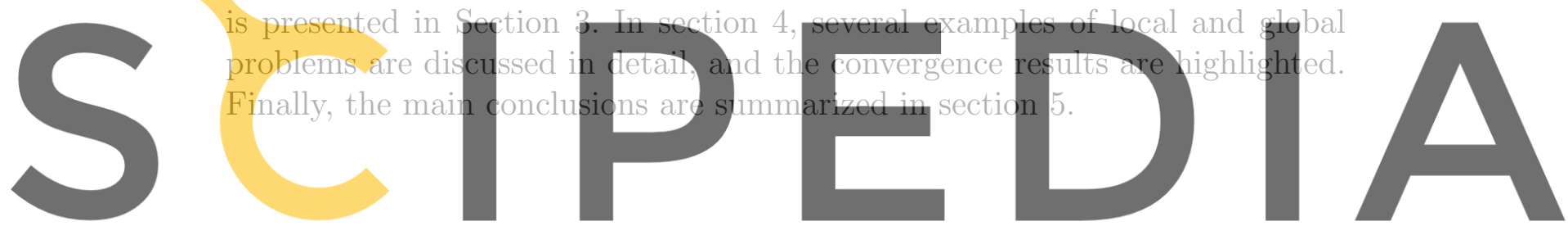

Register for free Peqteps/Atawtem.sentedia.com to download the version without the watermark

\subsection{Preliminaries}

Many elastoplastic models for small strains can be put in the general form [8]

$$
\begin{aligned}
\varepsilon & =\varepsilon^{e}+\varepsilon^{p} \\
\boldsymbol{\sigma} & =\mathbf{E} \varepsilon^{e} \\
\dot{\varepsilon}^{p} & =\dot{\lambda} \mathbf{m}(\boldsymbol{\sigma}, \boldsymbol{\kappa}) \\
\dot{\boldsymbol{\kappa}} & =\dot{\lambda} \mathbf{h}(\boldsymbol{\sigma}, \boldsymbol{\kappa}),
\end{aligned}
$$

where $\boldsymbol{\varepsilon}, \boldsymbol{\varepsilon}^{e}$ and $\boldsymbol{\varepsilon}^{p}$ are the total, elastic and plastic strain tensors respectively, $\boldsymbol{\sigma}$ is the Cauchy stress tensor, $\mathbf{E}$ is the elastic stiffness tensor, $\mathbf{m}$ is the flow vector, $\boldsymbol{\kappa}$ is the set of internal variables and $\mathbf{h}$ are the plastic moduli. The plastic multiplier $\dot{\lambda}$ is determined with the aid of the loading-unloading criterion, 
that can be expressed in Kuhn-Tucker form as [9]

$$
F(\boldsymbol{\sigma}, \boldsymbol{\kappa}) \leq 0 \quad \dot{\lambda} \geq 0 \quad F(\boldsymbol{\sigma}, \boldsymbol{\kappa}) \dot{\lambda}=0,
$$

where $F(\boldsymbol{\sigma}, \boldsymbol{\kappa})$ is the yield function that defines the admissible stress states.

\subsection{The MRS-Lade model}

The MRS-Lade model has been developed at the University of Colorado by Macari, Runesson and Sture [2] and it is a further development of Lade's three-invariant model for cohesionless soils. The model is used to simulate the behaviour of granular materials, such as sand, under both low and high confinement stresses [5,7]. It features 1) a two-surface yield function, comprising a smooth cone surface and a smooth cap surface intersecting in a plane curve (ellipse segment) in the deviatoric plane, 2) hardening and softening variables that depend on dissipated plastic work, and 3) a non-associated flow rule in the meridian plane of the cone region.

Several slight modifications to the original formulation of the model have been
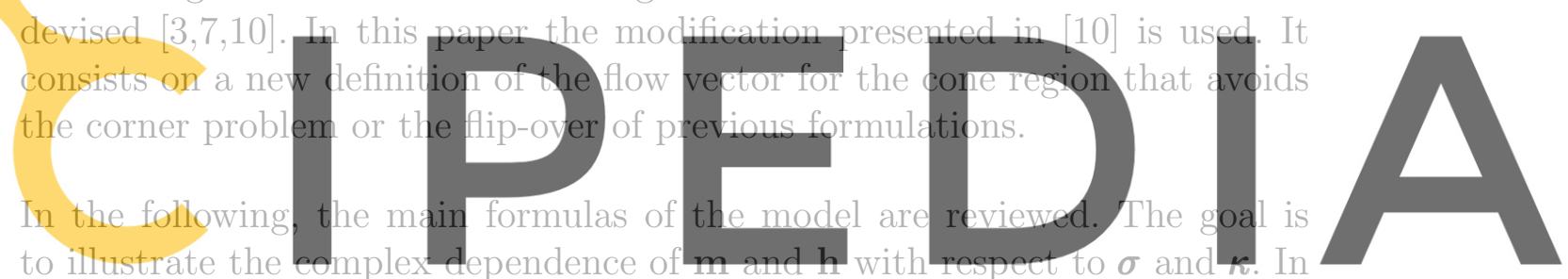

Appendix A, some additional formulas and the expanded expressions of $m$ and

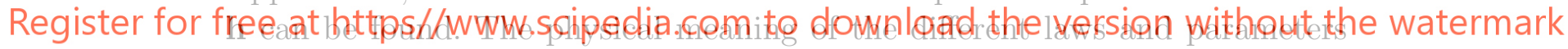
and the main characteristics of the model are widely discussed in [2].

The yield function $F$ depends on the three stress invariants ( $p, q$ and $\theta$, defined in Appendix A) and two internal variables, $\kappa_{\text {con }}$ and $\kappa_{\text {cap }}$. It is defined in two parts, the cone and the cap:

$$
\begin{gathered}
F_{\text {con }}\left(\boldsymbol{\sigma}, \kappa_{\text {con }}\right)=q\left(1+\frac{q}{q_{\mathrm{a}}}\right)^{m} g(\theta)-\eta_{\text {con }}\left(\kappa_{\text {con }}\right) p \\
F_{\text {cap }}\left(\boldsymbol{\sigma}, \kappa_{\text {con }}, \kappa_{\text {cap }}\right)=\left(\frac{p-p_{\mathrm{m}}}{p_{\mathrm{r}}}\right)^{2}+\left(\frac{q\left(1+q / q_{\mathrm{a}}\right)^{m} g(\theta)}{f_{\mathrm{r}}}\right)^{2}-1
\end{gathered}
$$

with

$$
\begin{aligned}
p_{\mathrm{m}} & =\alpha p_{\text {cap }}\left(\kappa_{\text {cap }}\right) \\
p_{\mathrm{r}} & =(1-\alpha) p_{\text {cap }}\left(\kappa_{\text {cap }}\right) \\
f_{\mathrm{r}} & =\alpha \eta_{\text {con }}\left(\kappa_{\text {con }}\right) p_{\text {cap }}\left(\kappa_{\text {cap }}\right),
\end{aligned}
$$


where $q_{\mathrm{a}}, m$ and $\alpha$ are parameters of the model, $g(\theta)$ is the Willam-Warnke function, $\eta_{\text {con }}\left(\kappa_{\text {con }}\right)$ is a hardening/softening function related with the friction angle, and $p_{\text {cap }}\left(\kappa_{\text {cap }}\right)$ is a hardening function related with the triaxial compression strength. The definitions of $g(\theta), \eta_{\text {con }}\left(\kappa_{\text {con }}\right)$ and $p_{\text {cap }}\left(\kappa_{\text {cap }}\right)$ are in Appendix A. The trace of $F_{\text {con }}$ and $F_{\text {cap }}$ in the meridian plane and in the deviatoric plane are depicted in Figure 1. The nonlinear shape of the hardening/softening function $\eta_{\text {con }}\left(\kappa_{\text {con }}\right)$ is shown in Figure 2.

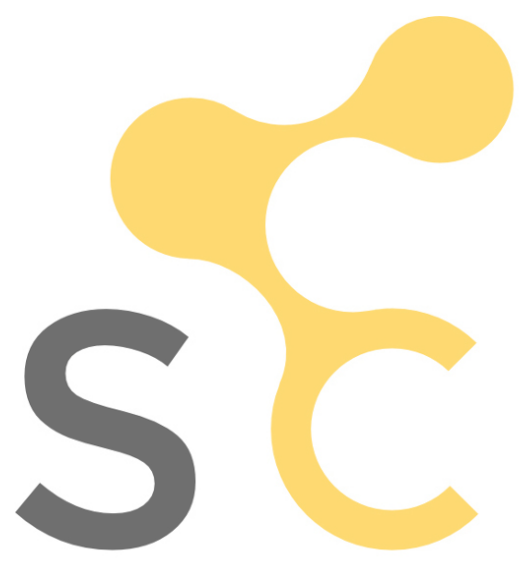

(a)
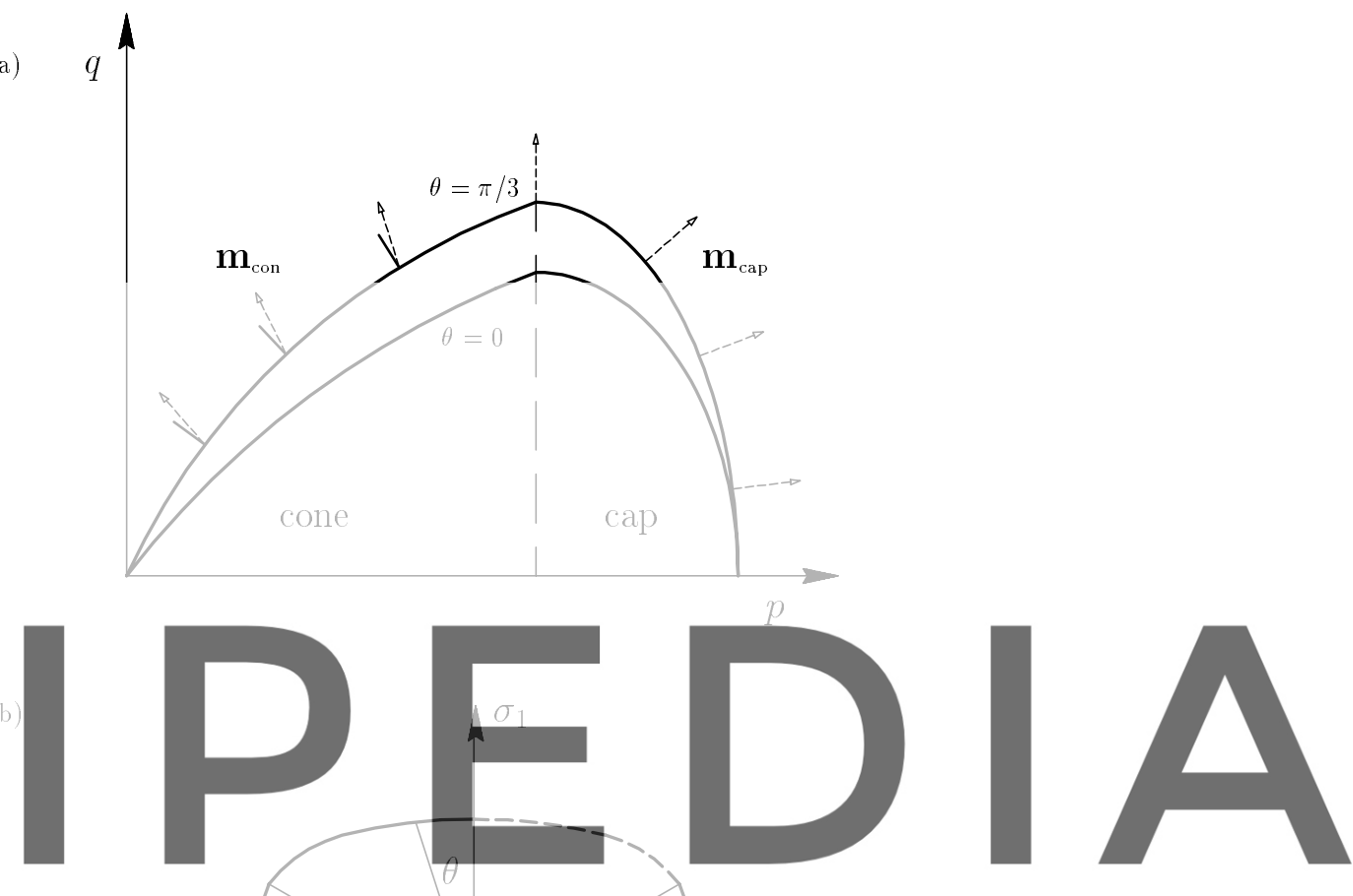

Register for free at https//www.scipedia.com to download the version without the watermark

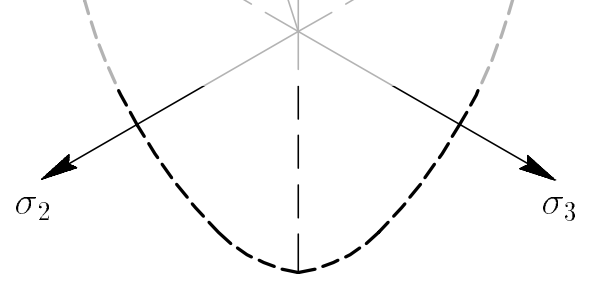

Figure 1. MRS-Lade model. Trace of the yield criterion: (a) on the meridian plane; (b) on the deviatoric plane

The plastic flow vector $\mathbf{m}$ is also defined in two parts, cone and cap:

$$
\mathbf{m}\left(\boldsymbol{\sigma}, \kappa_{\text {con }}, \kappa_{\text {cap }}\right)=\left\{\begin{array}{lll}
\mathbf{m}_{\text {con }}=\mathbf{A n}_{\text {con }} & \text { if } & p \leq \alpha p_{\text {cap }}\left(\kappa_{\text {cap }}\right) \\
\mathbf{m}_{\text {cap }}=\mathbf{n}_{\text {cap }} & \text { if } & p>\alpha p_{\text {cap }}\left(\kappa_{\text {cap }}\right)
\end{array}\right.
$$




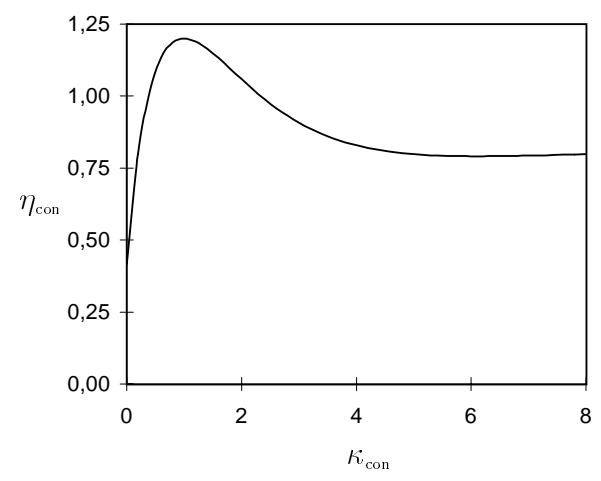

Figure 2. MRS-Lade model. Hardening/softening function $\eta_{\mathrm{con}}\left(\kappa_{\mathrm{con}}\right)$

with

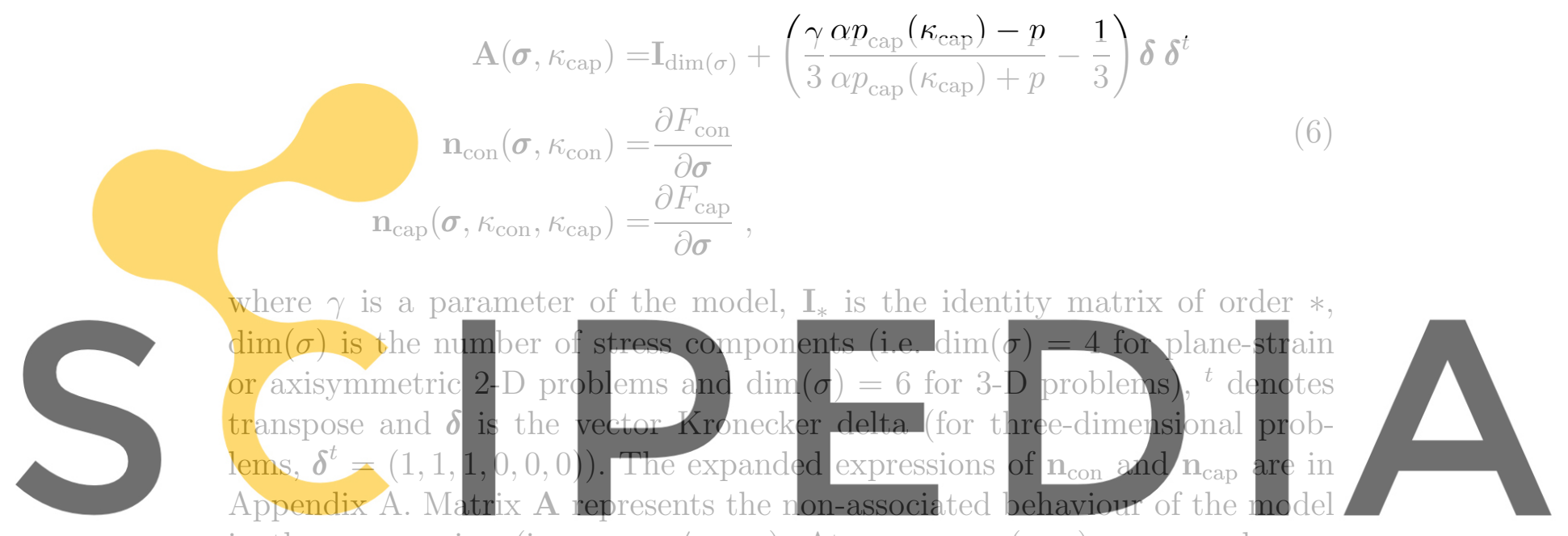

in the cone region (i.e. $\left.\mathbf{m}_{\text {con }} \neq \mathbf{n}_{\text {con }}\right)$. At $p=\alpha p_{\text {cap }}\left(\kappa_{\text {cap }}\right), \mathbf{m}_{\text {con }}$ and $\mathbf{m}_{\text {cap }}$

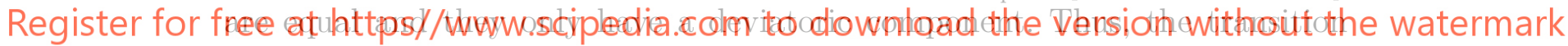

of the flow vector between cone and cap regions is smooth. In the original

formulation of the model [2], the matrix $\mathbf{A}$ is constant. As a consequence, the expression of $\mathbf{m}_{\text {con }}$ is a little simpler, but the transition of the flow vector at the cone-cap intersection is non-smooth and an expensive corner algorithm must be implemented to solve the local problem.

The evolution of the internal variables is defined as

$$
\begin{aligned}
\kappa_{\text {con }} & =\frac{1}{c_{\text {con }} p_{\mathrm{a}}}\left(\frac{p}{p_{\mathrm{a}}}\right)^{-l} \dot{w}^{p} \\
\kappa_{\text {cap }} & =\frac{1}{c_{\text {cap }} p_{\mathrm{a}}}\left(\frac{p_{\text {cap }, 0}}{p_{\text {a }}}\right)^{-r} \dot{w}^{p}
\end{aligned}
$$

where $c_{\text {con }}, c_{\text {cap }}, p_{\text {a }}, p_{\text {cap }, 0}, l$ and $r$ are parameters of the model and $\dot{w}^{p}$ is the rate of plastic work dissipated during loading along the stress path:

$$
\dot{w}^{p}=\boldsymbol{\sigma}^{t} \dot{\varepsilon}^{p}
$$


Combining equations (7), (8) and the last equation in (1) shows that the hardening moduli $\mathbf{h}=\left(h_{\text {con }}, h_{\text {cap }}\right)$ are

$$
\begin{aligned}
& h_{\text {con }}\left(\boldsymbol{\sigma}, \kappa_{\text {con }}, \kappa_{\text {cap }}\right)=\frac{1}{c_{\text {con }} p_{\mathrm{a}}}\left(\frac{p}{p_{\mathrm{a}}}\right)^{-l} \boldsymbol{\sigma}^{t} \mathbf{m} \\
& h_{\text {cap }}\left(\boldsymbol{\sigma}, \kappa_{\text {con }}, \kappa_{\text {cap }}\right)=\frac{1}{c_{\text {cap }} p_{\mathrm{a}}}\left(\frac{p_{\text {cap }, 0}}{p_{\text {a }}}\right)^{-r} \boldsymbol{\sigma}^{t} \mathbf{m} .
\end{aligned}
$$

Equations (5), (6) and (9) clearly exhibit a complex dependence of the flow vector $\mathbf{m}$ and the hardening moduli $\mathbf{h}$ with respect to the stresses $\boldsymbol{\sigma}$ and the internal variables $\boldsymbol{\kappa}$. This makes the efficient time-integration of the MRS-Lade model a challenging issue, as discussed in the following.

\section{Proposed approach}

\section{Numerical time-integration: the local and global problems}

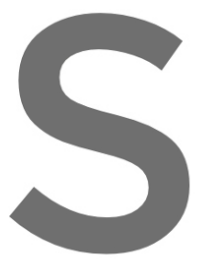

Time-integration
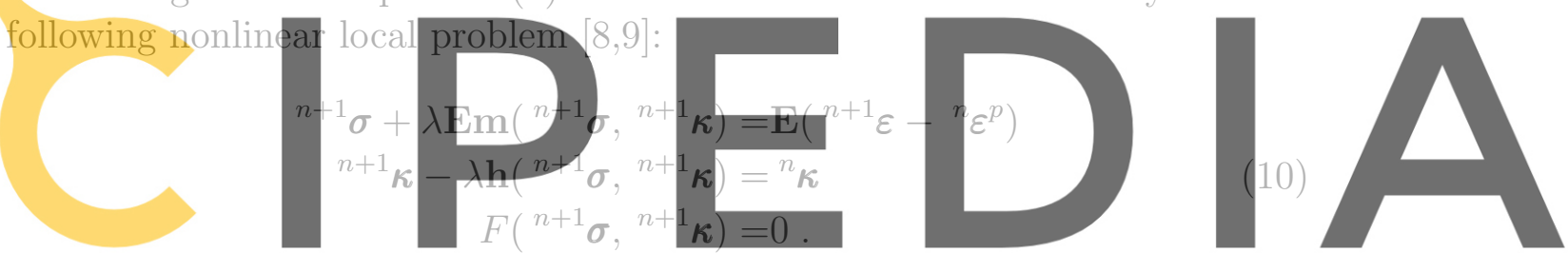

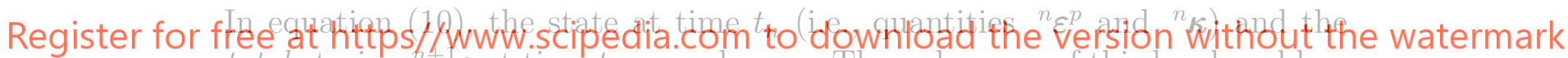
total strains ${ }^{-1} \varepsilon$ at time $t_{n+1}$ are known. The unknowns of this local problem are the stresses ${ }^{n+1} \sigma$ and the internal variables ${ }^{n+1} \kappa$ at time $t_{n+1}$, and the incremental plastic multiplier $\lambda$.

To solve this nonlinear local problem with the Newton-Raphson method the Jacobian of the residual is needed. Using standard vector notation of computational mechanics [11] and dropping the superscript $n+1$ the Jacobian can be written as

$$
\mathbf{J}=\left(\begin{array}{ccc}
\left(\mathbf{I}_{\operatorname{dim}(\sigma)}+\lambda \mathbf{E} \frac{\partial \mathbf{m}}{\partial \boldsymbol{\sigma}}\right) & \lambda \mathbf{E} \frac{\partial \mathbf{m}}{\partial \boldsymbol{\kappa}} & \mathbf{E m} \\
-\lambda \frac{\partial \mathbf{h}}{\partial \boldsymbol{\sigma}} & \left(\mathbf{I}_{\operatorname{dim}(\kappa)}-\lambda \frac{\partial \mathbf{h}}{\partial \boldsymbol{\kappa}}\right) & -\mathbf{h} \\
\mathbf{n}^{t} & \boldsymbol{\xi}^{t} & 0
\end{array}\right)
$$

where $\mathbf{n}$ and $\boldsymbol{\xi}$ are the derivatives of $F(\boldsymbol{\sigma}, \boldsymbol{\kappa})$ with respect to $\boldsymbol{\sigma}$ and $\boldsymbol{\kappa}$ respectively, and $\operatorname{dim}(\kappa)$ is the number internal variables (i.e. $\operatorname{dim}(\kappa)=2$ for the MRS-Lade model). 
On the other hand, to solve the global problem with quadratic convergence it is necessary to use the consistent tangent matrix $[4,12]$. To compute this matrix, the consistent tangent moduli $d^{n+1} \boldsymbol{\sigma} / d^{n+1} \varepsilon$ at each Gauss point are needed. They are obtained by linearizing equation (10). This linearization can be represented in a compact form as [13]

$$
\mathbf{P}^{t} \mathbf{J}^{-1} \mathbf{P E}
$$

where $\mathbf{P}^{t}=\left(\mathbf{I}_{\operatorname{dim}(\sigma)}, \mathbf{0}_{\operatorname{dim}(\kappa)+1}\right)$ is the projection matrix on stress space. Therefore, the Jacobian matrix, equation (11), is needed for both the local and the global problems.

The most difficult components to compute of the Jacobian are typically the derivatives of $\mathrm{m}$ and $\mathrm{h}$ with respect to $\sigma$ and $\kappa$ [1]. This is the case for the MRS-Lade model, which exhibits a high coupling of all the components. Note, for instance, that the hardening moduli $\mathbf{h}$ are defined in terms of the flow vector $\mathbf{m}$. This is caused by the fact that plastic work drives the hardening, see equation (7). Thus $\mathbf{h}$ depends on $\sigma$ and $\kappa$ both explicitly and through $\mathbf{m}$, see equation (9). This coupling makes the analytical computation of the derivatives a very cumbersome task. Jeremić and Sture, for instance, only
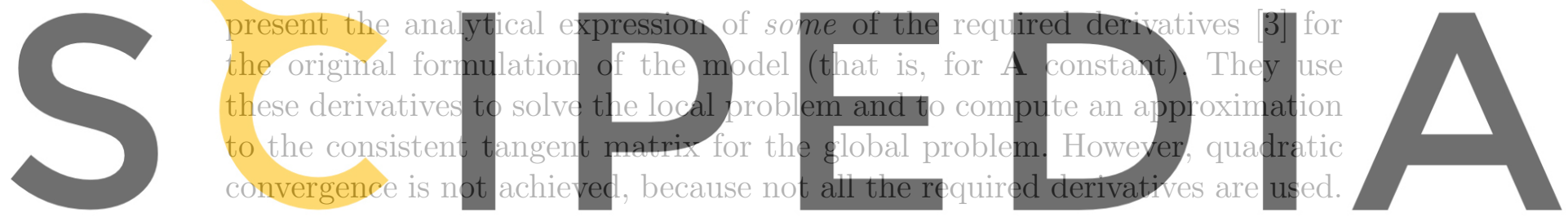

Register for free at https//www.scipedia.com to download the version without the watermark

\section{Numerical differentiation}

Indeed, quadratic convergence can only be attained by means of a full NewtonRaphson method. That is, all the derivatives of $\mathbf{m}$ and $\mathbf{h}$ with respect to $\boldsymbol{\sigma}$ and $\boldsymbol{\kappa}$ are needed. One possibility would be to obtain the analytical expression of the missing derivatives. However, this is rather involved, even with the help of an algebraic manipulator. For this reason, a different course is followed here [1]: all required derivatives are approximated numerically. Three of the techniques discussed in [1] will be employed: the forward difference scheme, $1 \mathrm{ND}-\mathrm{O}(h)$, the centered difference scheme, 1ND-O $\left(h^{2}\right)$, and the scheme based on complex variables, 1CND-O $\left(h^{2}\right)$, see Table 1 . The forward difference scheme is first-order accurate, and the other two schemes are second-order accurate. With these schemes, the derivative of $m_{i}$ with respect to $\kappa_{j}$ (recall that vector 
notation is used), for instance, is approximated either by

$$
\begin{aligned}
1 \mathrm{ND}-\mathrm{O}(h) & \frac{\partial m_{i}}{\partial \kappa_{j}}(\boldsymbol{\sigma}, \boldsymbol{\kappa})=\frac{m_{i}\left(\boldsymbol{\sigma}, \boldsymbol{\kappa}+h \mathbf{e}_{j}\right)-m_{i}(\boldsymbol{\sigma}, \boldsymbol{\kappa})}{h}, \\
1 \mathrm{ND}-\mathrm{O}\left(h^{2}\right) & \frac{\partial m_{i}}{\partial \kappa_{j}}(\boldsymbol{\sigma}, \boldsymbol{\kappa})=\frac{m_{i}\left(\boldsymbol{\sigma}, \boldsymbol{\kappa}+h \mathbf{e}_{j}\right)-m_{i}\left(\boldsymbol{\sigma}, \boldsymbol{\kappa}-h \mathbf{e}_{j}\right)}{2 h}, \\
1 \mathrm{CND}-\mathrm{O}\left(h^{2}\right) & \frac{\partial m_{i}}{\partial \kappa_{j}}(\boldsymbol{\sigma}, \boldsymbol{\kappa})=\frac{\operatorname{Im}\left(m_{i}\left(\boldsymbol{\sigma}, \boldsymbol{\kappa}+\sqrt{-1} h \mathbf{e}_{j}\right)\right)}{h},
\end{aligned}
$$

where $h$ is the stepsize and $\mathbf{e}_{j}$ is the $j$ th unit vector. Similar expressions are used for $\partial \mathbf{m} / \partial \boldsymbol{\sigma}, \partial \mathbf{h} / \partial \boldsymbol{\sigma}$ and $\partial \mathbf{h} / \partial \boldsymbol{\kappa}$. The approximated derivatives are then used to solve the local and the global problems.

\begin{tabular}{|l|l|}
\hline Notation & Description \\
\hline 1 ND-O $(h)$ & Forward difference scheme $\left(1^{\text {st }}\right.$ order accurate $)$ \\
\hline 1 ND-O $\left(h^{2}\right)$ & Centered difference scheme $\left(1^{\text {st }}\right.$ order accurate $)$ \\
\hline 1 CND-O $\left(h^{2}\right)$ & Approximation based on complex variables (2 $2^{\text {nd }}$ order accurate) \\
\hline
\end{tabular}

\section{Table 1}

Numerical approximations to first derivatives
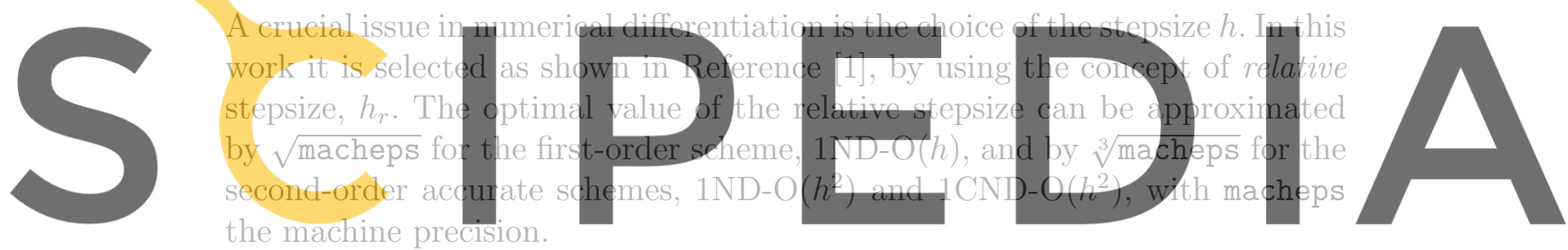

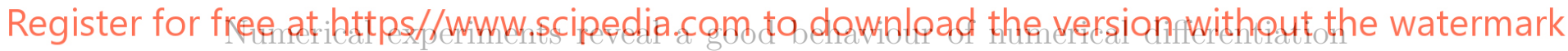

(that is, quadratic convergence for both the local and the global problems) for

a wide range of relative stepsizes, $h_{r}$. In order to reduce the effect of rounding errors, $h_{r}$ is taken as a negative power of $2\left(h_{r}=2^{-k}\right)$, not of $10\left(h_{r}=10^{-k}\right)$. This choice is relevant in some critical zones, as illustrated in next section.

\section{$5 \quad$ Examples}

In this section, several local and global problems are solved quadratically with numerical differentiation. The three techniques of Table 1 are compared and the main features of each one are remarked.

Two sets of parameters have been used, see Table 2. Soil S1 is a dense Sacramento River sand [7]. Soil S2 is a small modification of soil S1. The modifications are 1) a smaller value of $\eta_{\text {con }}^{-}$, which reduces the size of the elastic 
domain ( $\eta_{\text {con }}^{-}$is the maximum value of $\eta_{\text {con }}$, see equations (A.4) and (A.5) in appendix $\mathrm{A}$ ), and 2) different values of $c_{\mathrm{con}}$ and $\epsilon$, which result in a more nonlinear evolution of the cone internal variable, $\kappa_{\text {con }}$, see equations (9), (A.4) and (A.5). With these two modifications, soil S2 is quite more demanding from a numerical point of view than soil S1.

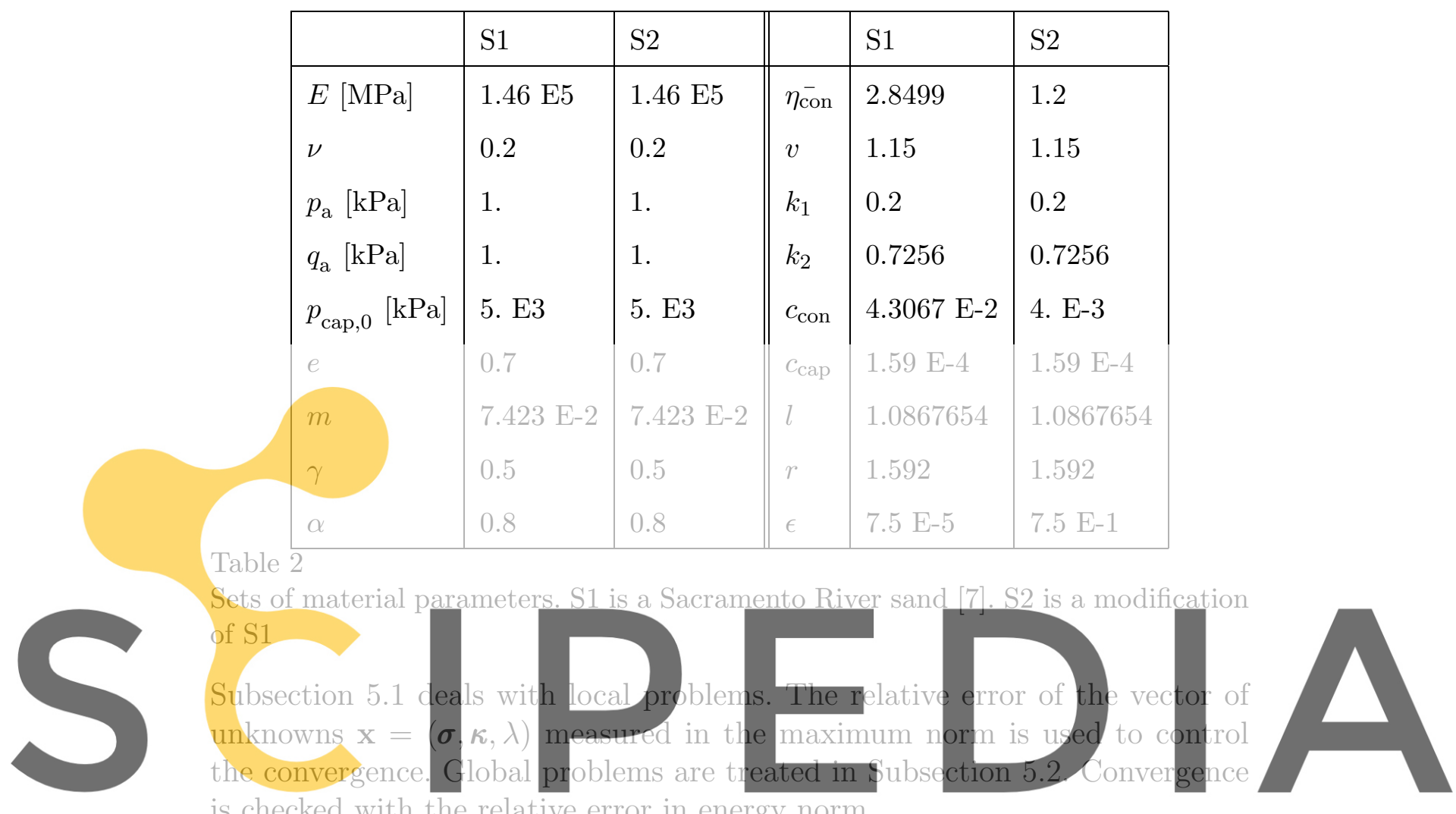

is checked with the relative error in energy norm.

Register for free at https//www.scipedia.com to download the version without the watermark All the computations (except where the opposite is explicitly stated) have been performed by using a negative power of 2 , not of 10 , as the relative stepsize $\left(h_{r}=2^{-k}\right)$, to reduce the effect of rounding errors. However, relative stepsizes are expressed as powers of 10 to indicate clearly the order of magnitude. For instance, $h_{r}=10^{-6}$ in the text or in a table means that the actual computation is performed with $h_{r}=2^{-19}$.

Strict tolerances have been used: $10^{-14}$ for local problems and $10^{-8}$ to $10^{-10}$ for global problems. This allows for a comparative assessment of the three differentiation techniques. Quite larger values may be chosen in practice.

\subsection{Local problems}

In the local problem, numerical differentiation is applied to compute the Jacobian shown in equation (11) at each Gauss point. 
In order to show that quadratic convergence is obtained in all stress-internal variable space, three different deformation paths are considered. The paths are characterized by an initial stress-internal variable state, $\boldsymbol{\sigma}_{\text {ini }}$ and $\boldsymbol{\kappa}_{\text {ini }}$, and a total strain increment, $\Delta \boldsymbol{\varepsilon}$ (applied in 50 steps) see Table 3 . The material parameters of soil S2 have been used.

\begin{tabular}{|l|l|l|l|}
\hline & Path A & Path B & Path C \\
\hline$\sigma_{\text {ini }}$ & $(1000,1000,1000,0)$ & $(4800,4800,4800,0)$ & $(4800,4800,4800,0)$ \\
$\boldsymbol{\kappa}_{\text {ini }}$ & $(0,0)$ & $(0,0)$ & $(0,0)$ \\
$\Delta \varepsilon$ & $(0,0,0,0.2)$ & $(0,0,0,0.2)$ & $(-0.1,0,0,0)$ \\
$n$ & 50 & 50 & 50 \\
\hline
\end{tabular}

Table 3

Definition of the three stress paths for the local problems

In Figure 3 the trace on the meridian plane of the three stress paths and the trace of the initial and final yield criteria are depicted. Paths A and B correspond to pure shear deformation, see $\Delta \varepsilon$ in Table 3. Path A develops in the cone region, and path $\mathrm{B}$ starts in the cap region and then changes to the cone region. Path $\mathrm{C}$ corresponds to uniaxial compression, see Table 3 , and it develops in the cap region. In Table 4 the evolution of the Lode angle, $\theta$, is shown. Note that, in general, the paths are three-dimensional curves in the three-invariant space $(p, q, \theta)$. Indeed, $\theta$ changes during loading in paths $\mathrm{A}$ and $\mathrm{B}$. For path $\mathrm{C}$, on the other hand, $\theta$ remains constant and equal to $\pi / 3$. Finally, note that the three paths start in hardening regime and finish during softening. Therefore, a wide range of different local problems is covered.

Table 4

\begin{tabular}{|l|c|c|c|c|c|}
\hline Step & 1 & 5 & 10 & 25 & 50 \\
\hline Path A & 40.3 & 51.5 & 53.1 & 54.1 & 53.9 \\
\hline Path B & 30.0 & 35.8 & 42.2 & 54.7 & 54.1 \\
\hline Path C & 60.0 & 60.0 & 60.0 & 60.0 & 60.0 \\
\hline
\end{tabular}

Evolution of the Lode angle $\theta$ (in degrees) during the three stress paths defined in Table 3

In the following, quadratic convergence results are presented and analyzed for moderate strain increments. After that, the behaviour of the three numerical differentiation schemes, see Table 1, is compared. Finally, quadratic convergence results for large excursions outside the elastic domain are also shown.

\subsubsection{Convergence illustration}

The convergence results for different steps of the three stress paths are depicted in Figure 4. These results correspond to local problems in the cone and the cap 

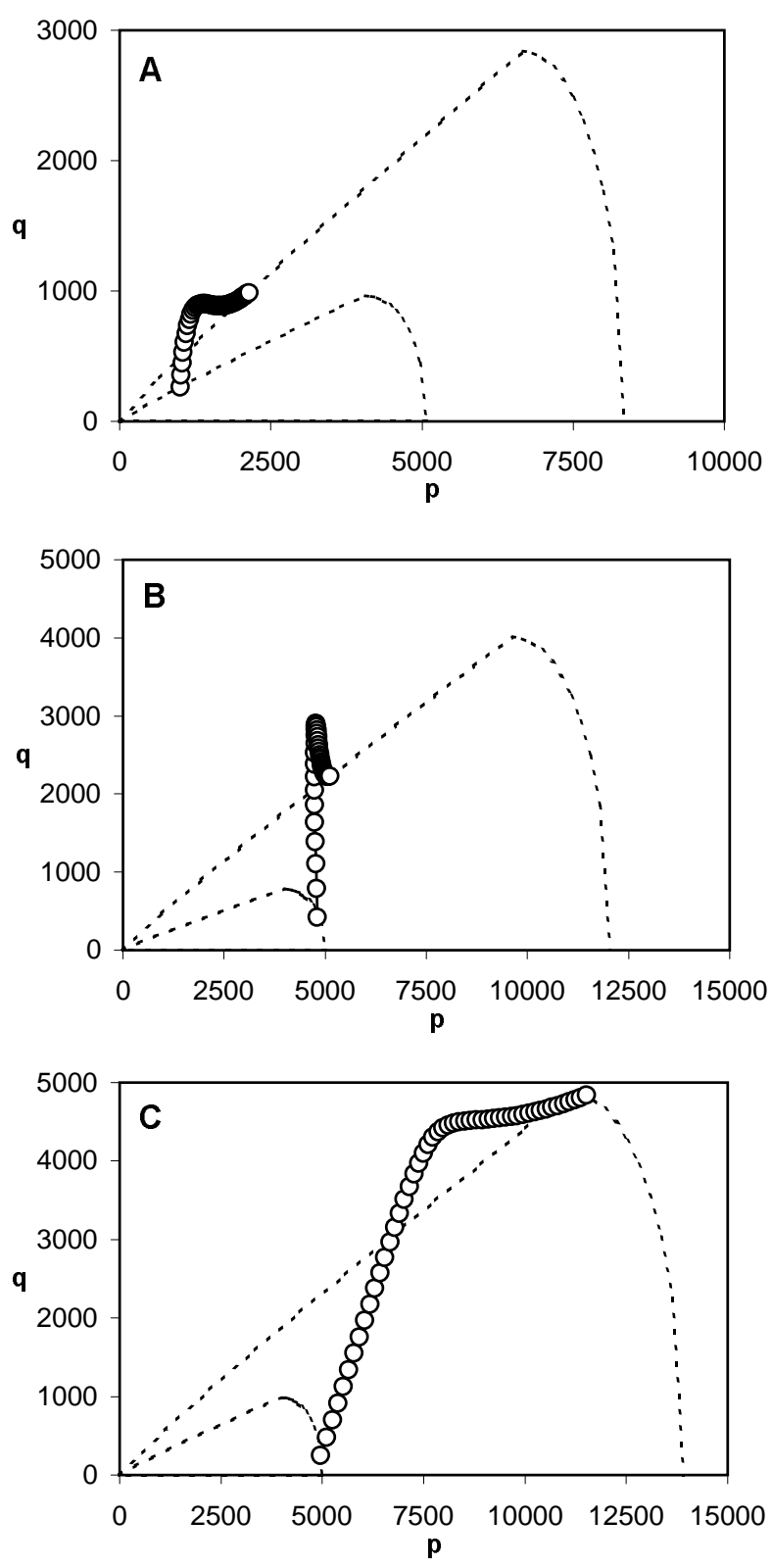

Figure 3. Trace of the three paths and of their initial and final yield criteria on the meridian plane

regions and to hardening and softening regimes. All the convergence results are quadratic up to a (very strict) tolerance of $10^{-14}$. These results have been obtained with the approximation $1 \mathrm{ND}-\mathrm{O}(h)$ and with $h_{r}=10^{-6}$, see equation (13) and Table 1 . The convergence is also quadratic if checked independently for each unknown of the local problem $(\boldsymbol{\sigma}, \boldsymbol{\kappa}$ and $\lambda)$, see Figure 5.

In Figure 6 the stress invariants and the yield criterion during the iterations are depicted. In three iterations the approximations are very close to the final result. The remaining iterations are just to improve the accuracy. 

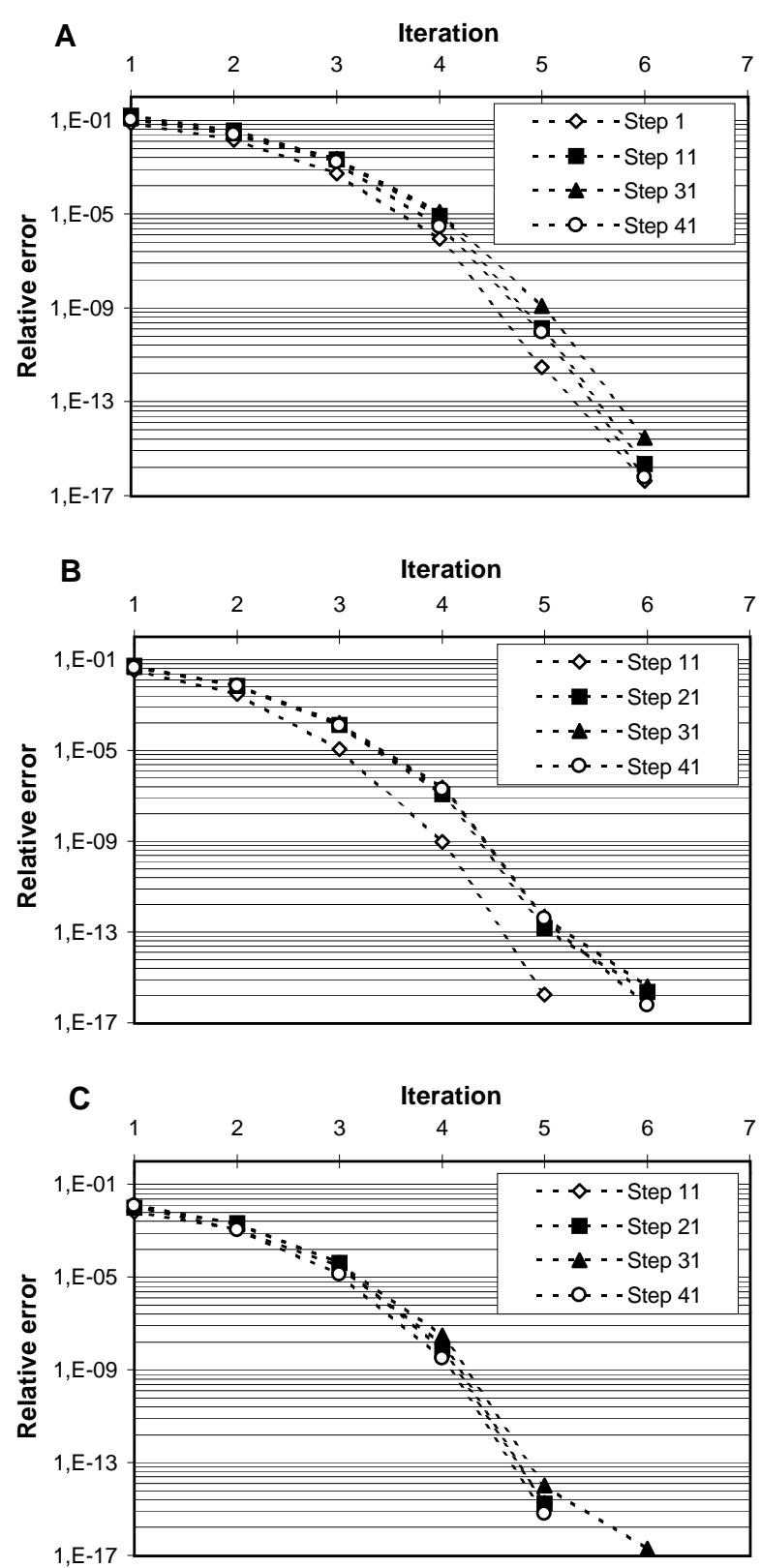

Figure 4. Convergence results for various steps of paths A, B and C

\subsubsection{Comparison of numerical differentiation schemes}

The three numerical differentiation schemes of Table 1 have been compared through the integration of paths A, B and C defined in Table 3.

The convergence results of step 31 of path A with different relative stepsizes $h_{r}$ are depicted in Figure 7. They are quadratic up to a tolerance of $10^{-14}$ for a wide range of $h_{r}$ with the three schemes. The same results are obtained with the other steps of paths A and B. In Table 5 , the ranges of $h_{r}$ that give 


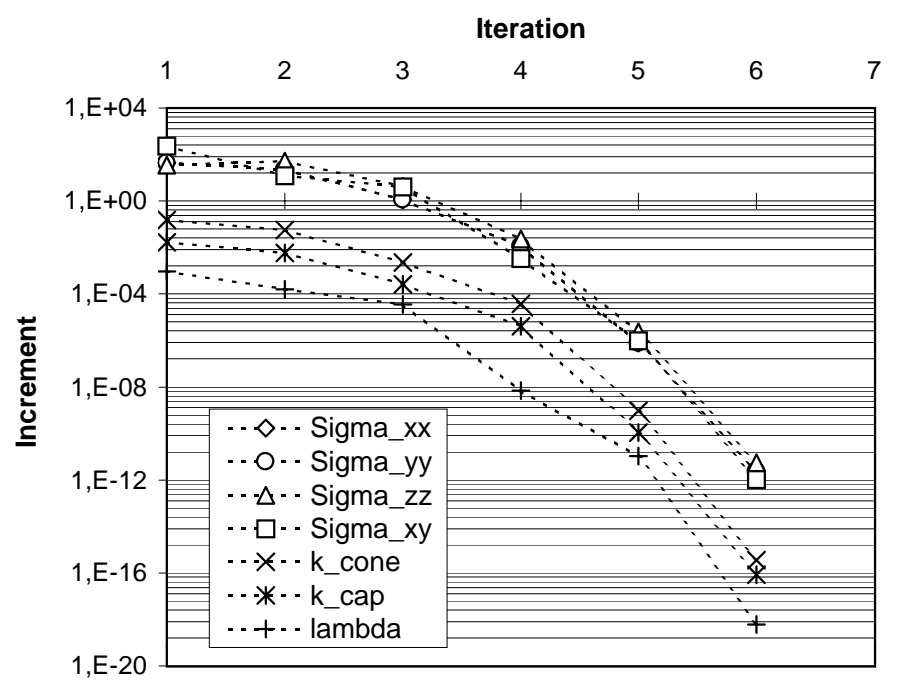

Figure 5. Convergence of the different unknowns of the local problem for step 31 of path A

quadratic convergence during all the steps of paths A and B are summarized. The main difference between the three techniques is that second order of accuracy provides quadratic convergence with larger $h_{r}$. This is in agreement with [1] and it is due to the fact that the truncation error of the second-order schemes is lower than for the first-order scheme.

The ranges of $h_{r}$ that give quadratic convergence during all the steps of path $\mathrm{C}$ are summarized in Table 6 . Both the ranges obtained using $h_{r}=10^{-k}$ and $h_{r}=2^{-k}$ are indicated. Two aspects are important: first, the ranges are quite narrower than for paths $\mathrm{A}$ and $\mathrm{B}$; and second, with the approximation 1ND$\mathrm{O}(h)$ the improvement of using $h_{r}=2^{-k}$ is notorious. This is because path $\mathrm{C}$ develops at Lode angle equal to $\pi / 3$. In this zone, the influence of the rounding errors is quite more important than in the other regions of the stress space. Nevertheless, the range in which one can choose $h_{r}$ is still wide enough and includes the approximation indicated before.

Table 5

\begin{tabular}{|l|c|c|}
\hline Num. approx. & Path A & Path B \\
\hline $1 \mathrm{ND}-\mathrm{O}(h)$ & $10^{-6}-10^{-9}$ & $10^{-6}-10^{-10}$ \\
\hline $1 \mathrm{ND}-\mathrm{O}\left(h^{2}\right)$ & $10^{-4}-10^{-10}$ & $10^{-4}-10^{-10}$ \\
\hline $1 \mathrm{CND}-\mathrm{O}\left(h^{2}\right)$ & $10^{-4}-10^{-11}$ & $10^{-4}-10^{-11}$ \\
\hline
\end{tabular}

Range of relative stepsizes $h_{r}$ that give quadratic convergence in the local problem, stress paths A and B 

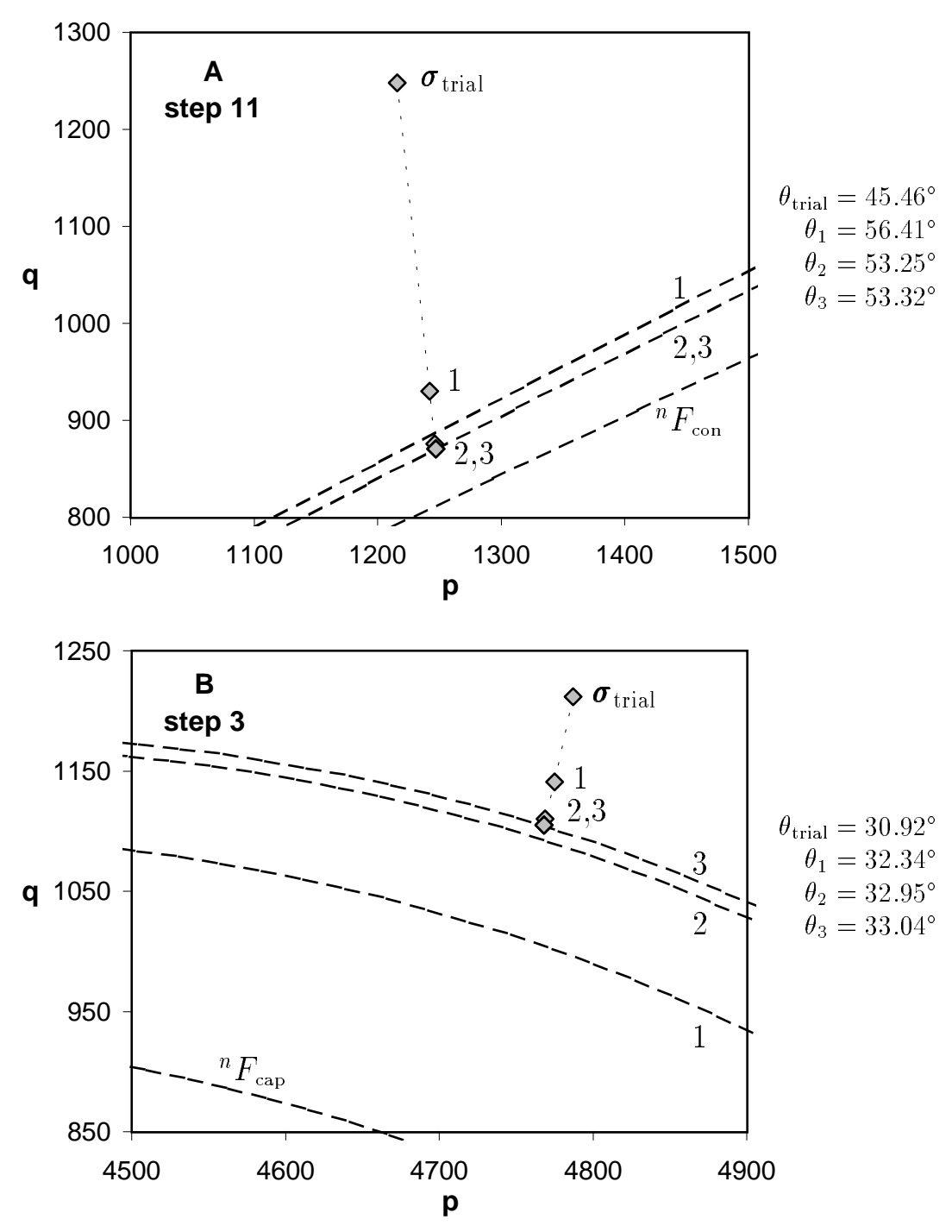

Figure 6. Evolution of the stress invariants and the yield criterion during the iterations of step 11 of path A and step 3 of path B

Table 6

\begin{tabular}{|l|c|c|}
\hline Num. approx. & $h_{r}=10^{-k}$ & $h_{r}=2^{-k}$ \\
\hline $1 \mathrm{ND}-\mathrm{O}(h)$ & $10^{-5}-10^{-6}$ & $10^{-5}-10^{-8}$ \\
\hline $1 \mathrm{ND}-\mathrm{O}\left(h^{2}\right)$ & $10^{-3}-10^{-7}$ & $10^{-3}-10^{-7}$ \\
\hline $1 \mathrm{CND}-\mathrm{O}\left(h^{2}\right)$ & $10^{-4}-10^{-9}$ & $10^{-4}-10^{-9}$ \\
\hline
\end{tabular}

Range of relative stepsizes $h_{r}$ that give quadratic convergence in the local problem, stress path $\mathrm{C}$

\subsubsection{Large excursions outside the elastic domain}

In order to show that quadratic convergence is also attained for large excursions outside the elastic domain, path A defined in Table 3 is solved with only 

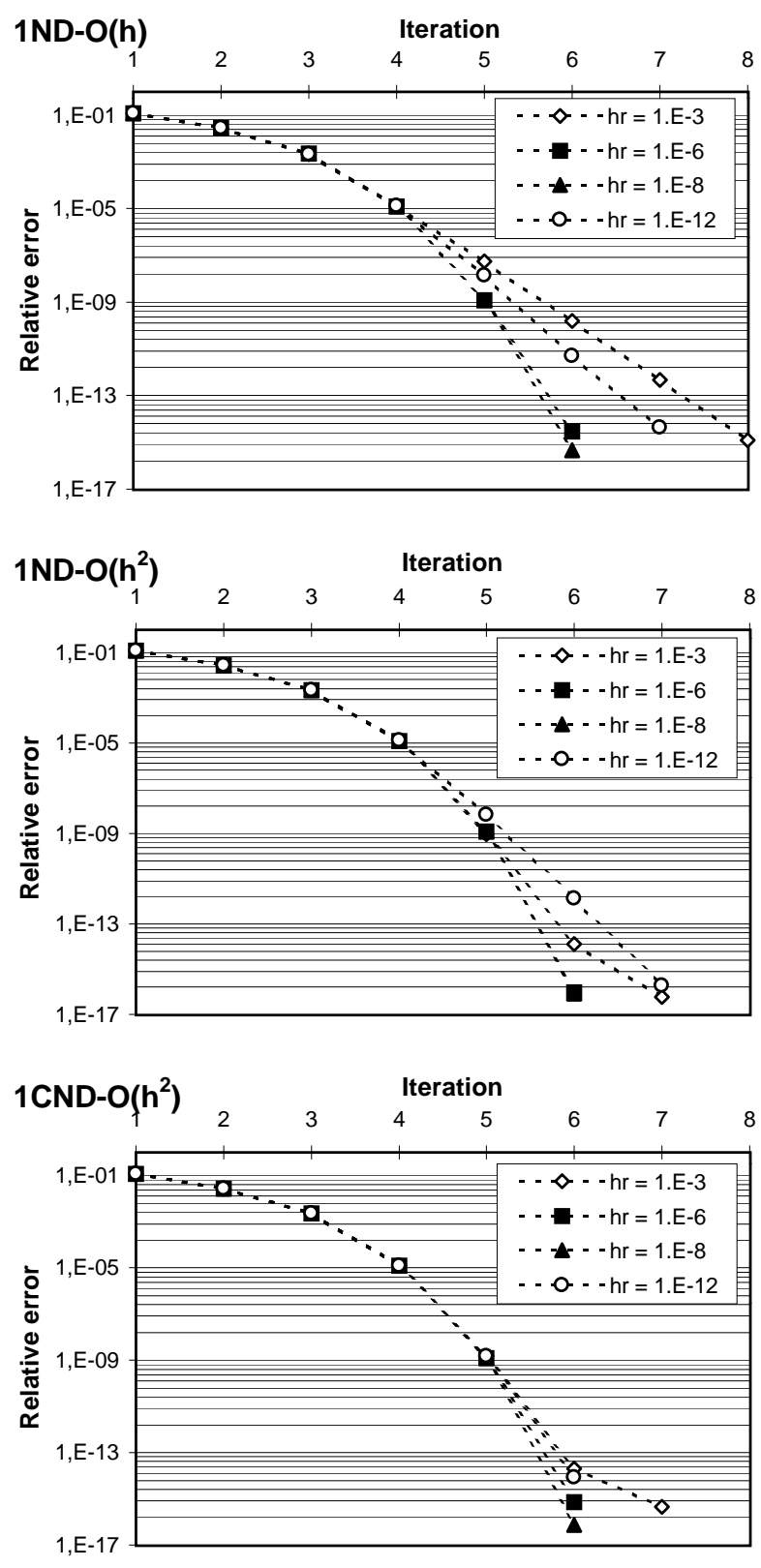

Figure 7. Convergence results for step 31 of path A using the approximations defined in Table 1 with several relative stepsizes $h_{r}$

10 steps. The convergence results for various steps, depicted in Figure 8, are again quadratic. On the other hand, note that, as expected, more iterations than in the original integration of path A with 50 steps are needed (compare Figures 8 and 4). This is clearly due to the use of the solution of one step as the initial approximation for the next step. Larger steps mean worse initial approximations and, thus, more iterations.

To summarize this subsection on local problems: quadratic convergence can 


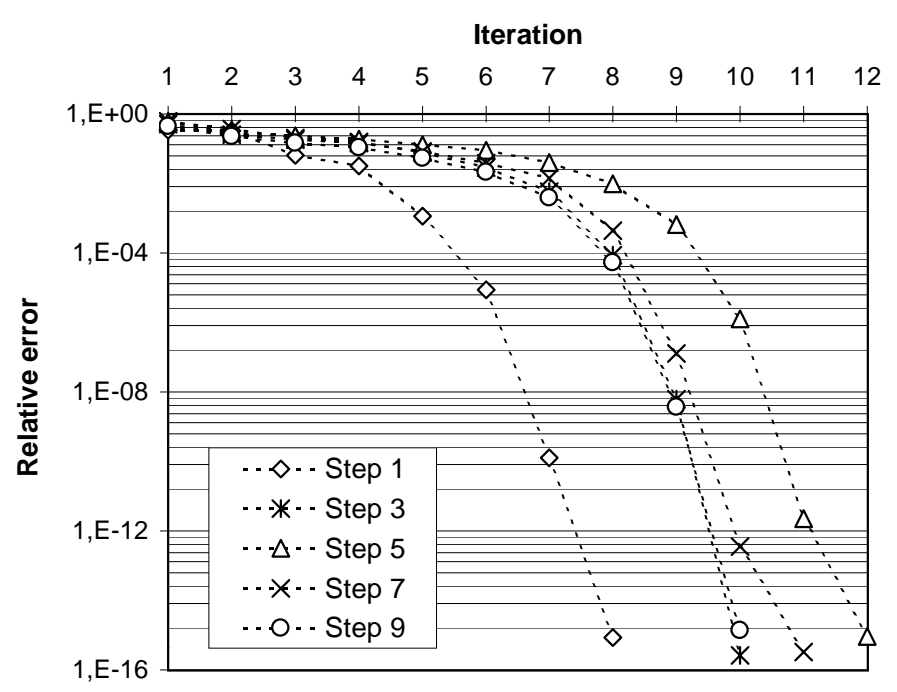

Figure 8. Convergence results for path A with only 10 steps

be attained in a simple manner with any of the three techniques of numerical differentiation. This is valid for any stress path (cone and cap regions, hardening and softening regimes), and for both moderate and large steps. The choice of the stepsize presents no difficulties, because quadratic convergence is obtained for a wide range of relative stepsizes.

\subsection{Global problems}

In this subsection, numerical differentiation is applied to solve several boundary value problems (i.e., global problems). That is, the numerical approximations of Table 1 are employed to compute consistent tangent matrices, see equation (12). Moreover, they are also used to solve the corresponding local problems.

Three examples are presented: the vertical displacement of a pile, a triaxial test with an homogeneous sample and a triaxial test with a non-homogeneous sample. These examples illustrate that the three numerical approximations to first derivatives of the flow vector and the hardening moduli, see Table 1, are useful to solve the global problem with quadratic convergence. Moreover, their main features (range of adequate relative stepsizes and computational cost) are compared.

\subsubsection{Vertical displacement of a pile}

The first example is the vertical displacement of a pile. The definition of the problem is presented by Potts and Gens [14], and it is only summarized here. 
Figure 9 shows the finite element mesh. It corresponds to a horizontal disc of soil. The thickness of the disc is $5 \mathrm{~cm}$ and the pile radius is $7.5 \mathrm{~cm}$. A hydrostatic initial stress state of $-250 \mathrm{kPa}$ is imposed. To model the loading of the pile, a vertical displacement of $0.625 \mathrm{~cm}$ is prescribed over the boundary AF in 25 load steps. To model the infinite extension of the disc, zero vertical displacements are prescribed over the boundary CD. Due to the essentially one-dimensional nature of the problem, vertical lines (such as EB) are prescribed to remain vertical during loading. The material parameters correspond to the dense Sacramento River sand, see Table 2.

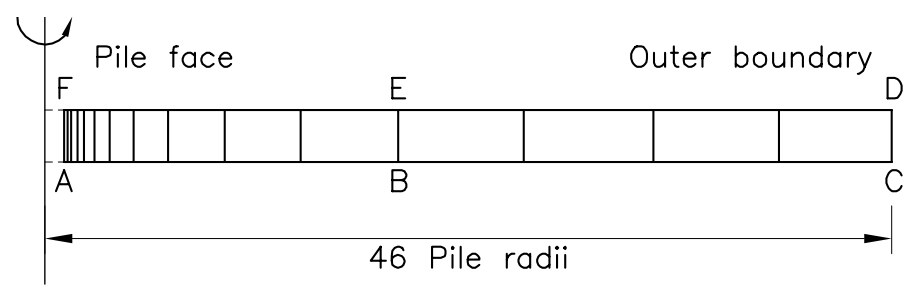

Figure 9. Pile problem (after Potts and Gens, 1985)

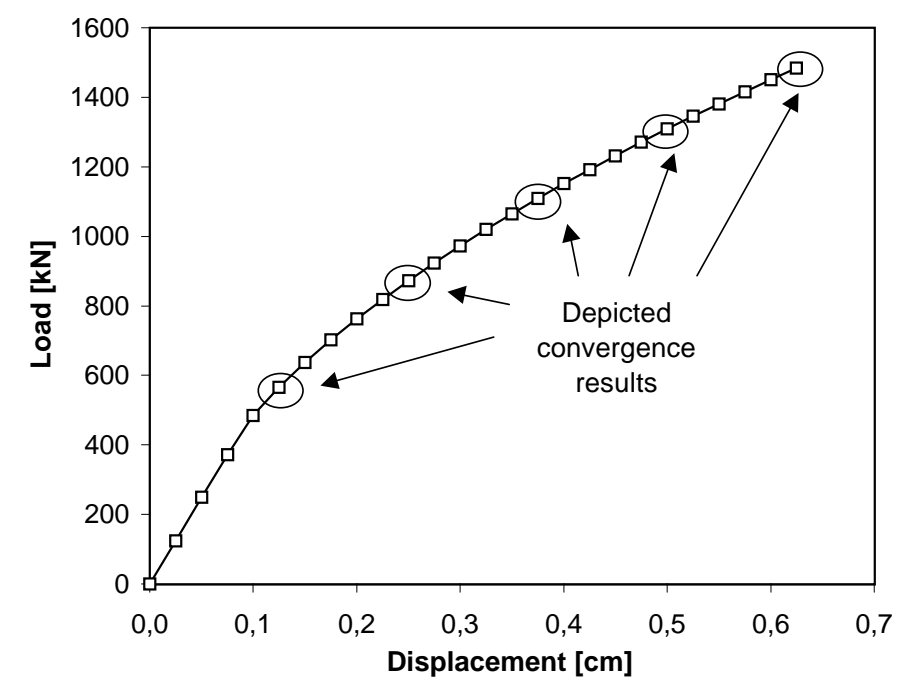

Figure 10. Load versus displacement curve for the pile problem

The load versus displacement curve is depicted in Figure 10. The most stressed points are those next to the boundary AF. Because of the one-dimensional behaviour of the problem, the limit state is reached when the integration points close to AF start the softening regime. After that, stresses are no longer transferred to the rest of the disc. Thus, this simple example only tests the behaviour during hardening. The convergence results for several load steps (indicated in Figure 10) are shown in Figure 11. Convergence is quadratic up to a strict tolerance of $10^{-10}$. These particular results have been obtained with the approximation 1CND-O $\left(h^{2}\right)$ and with $h_{r}=10^{-5}$. However, similar results are obtained with the other techniques and other relative stepsizes. In Table 
7, the ranges of $h_{r}$ that give quadratic convergence during all the test are summarized.

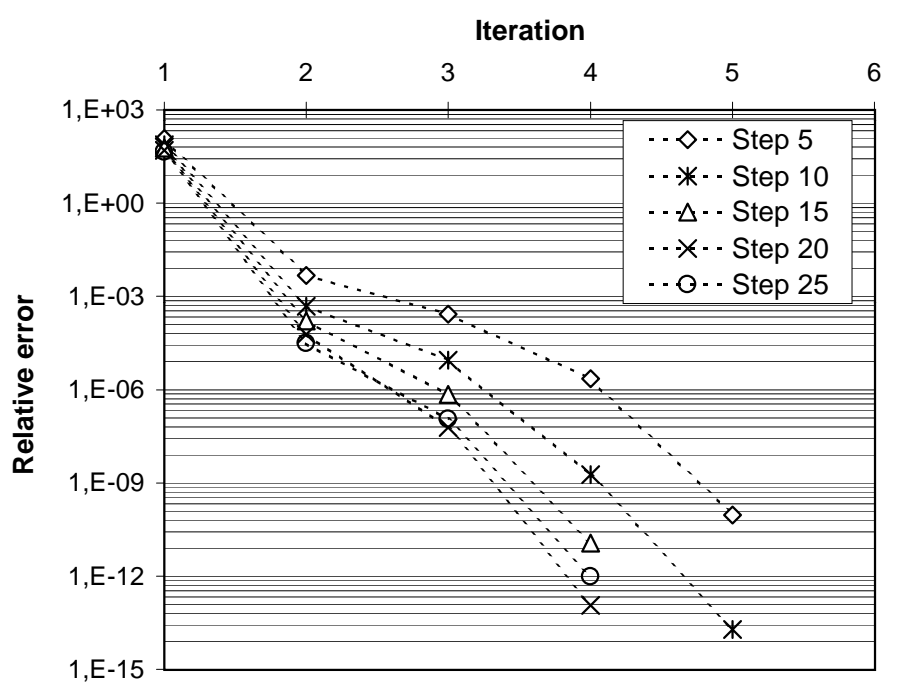

Figure 11. Convergence results for various load steps of the pile problem

Table 7

\begin{tabular}{|l|l|}
\hline Num. approx. & Range of $h_{r}$ \\
\hline $1 \mathrm{ND}-\mathrm{O}(h)$ & $10^{-5}-10^{-9}$ \\
\hline $1 \mathrm{ND}-\mathrm{O}\left(h^{2}\right)$ & $10^{-3}-10^{-9}$ \\
\hline $1 \mathrm{CND}-\mathrm{O}\left(h^{2}\right)$ & $10^{-3}-10^{-9}$ \\
\hline
\end{tabular}

Range of relative stepsizes $h_{r}$ that give quadratic convergence up to a tolerance of $10^{-10}$ in the pile problem

In Table 8 the computational cost (CPU time) of the three techniques with several $h_{r}$ is summarized. The values are given in \% with respect to 1ND$\mathrm{O}(h)$ with $h_{r}=10^{-8}$. The number of iterations during all the load process is equal for all the entries in Table 8, except for $1 \mathrm{ND}-\mathrm{O}(h)$ with $h_{r}=10^{-3}$ and $10^{-4}$ (which require 6 and 1 extra iterations respectively). The results are quite independent of $h_{r}$ and are mainly related with the derivation technique. The 1ND-O $\left(h^{2}\right)$ and 1CND-O $\left(h^{2}\right)$ approximations are respectively $20 \%$ and $40 \%$ more expensive than the $1 \mathrm{ND}-\mathrm{O}(h)$ approximation. These results are in agreement with the fact that the cost of a complex function evaluation can be approximated (when additions and products are balanced) by the cost of four real function evaluations. Thus, the overhead of complex approximation (one complex evaluation of the flow vector and the hardening moduli) is twice that of centered differences (that needs two real evaluations).

Quadratic convergence results have been obtained without difficulties in a simple global problem. There are not significative differences between the convergence results of the three techniques. The ranges of optimal relative stepsize 


\begin{tabular}{|c|c|c|c|c|c|c|c|}
\hline$h_{r}$ & $10^{-3}$ & $10^{-4}$ & $10^{-5}$ & $10^{-6}$ & $10^{-7}$ & $10^{-8}$ & $10^{-9}$ \\
\hline $1 \mathrm{ND}-\mathrm{O}(h)$ & $113 \%$ & $106 \%$ & $107 \%$ & $101 \%$ & $101 \%$ & $100 \%$ & $101 \%$ \\
$1 \mathrm{ND}-\mathrm{O}\left(h^{2}\right)$ & $122 \%$ & $122 \%$ & $124 \%$ & $124 \%$ & $122 \%$ & $122 \%$ & $122 \%$ \\
$1 \mathrm{CND}-\mathrm{O}\left(h^{2}\right)$ & $141 \%$ & $143 \%$ & $139 \%$ & $139 \%$ & $139 \%$ & $140 \%$ & $138 \%$ \\
\hline
\end{tabular}

Table 8

Relative CPU time of the three numerical differentiation techniques with several relative stepsizes $h_{r}$ in the pile problem

are wide in all cases. Differences appear regarding computational cost.

\subsubsection{Triaxial test: homogeneous sample}

The second example is the triaxial test with an homogeneous sample. The material parameters, defined in Table 2, correspond to a Sacramento River sand. These parameters are the same that Macari et al. [7] used to simulate the triaxial test at Gauss point level. Note that $\epsilon$ is not defined in that reference, so an inverse problem has been solved to determine its value. Here, the triaxial test is solved at the global level, and, as expected, the results are homogeneous and equal to those presented in [7].

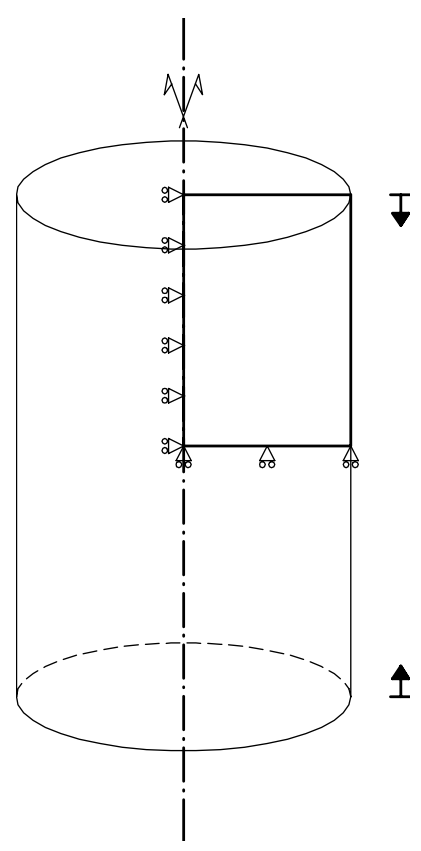

Figure 12. Triaxial test. Problem statement

The definition of the problem is summarized in Figure 12. The sample is axisymmetric. The top and bottom are assumed perfectly smooth and the vertical displacement is imposed simultaneously in both faces. Thus, just a half of the sample is considered in the numerical simulation. The load is divided in 
two phases: first the sample is precompressed, and second a vertical displacement is imposed at the top. A structured mesh of $150(10 \times 15)$ elements has been used. In Figure 13, the relationship between $q$ and the axial strain for several precompressions is depicted. They coincide with those presented in [7].

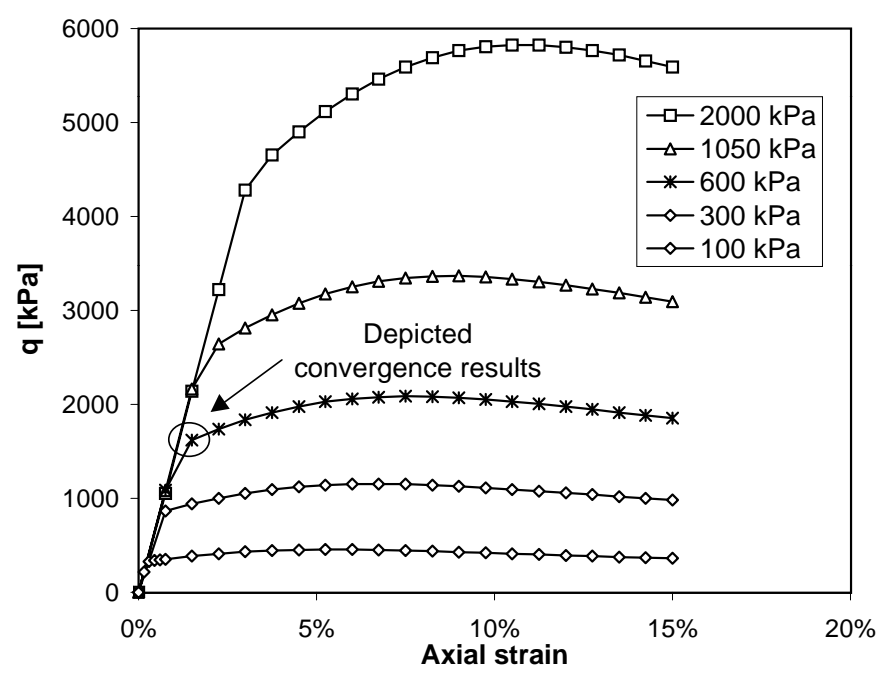

Figure 13. Invariant $q$ versus axial strain curves for the homogeneous triaxial problem with various precompressions

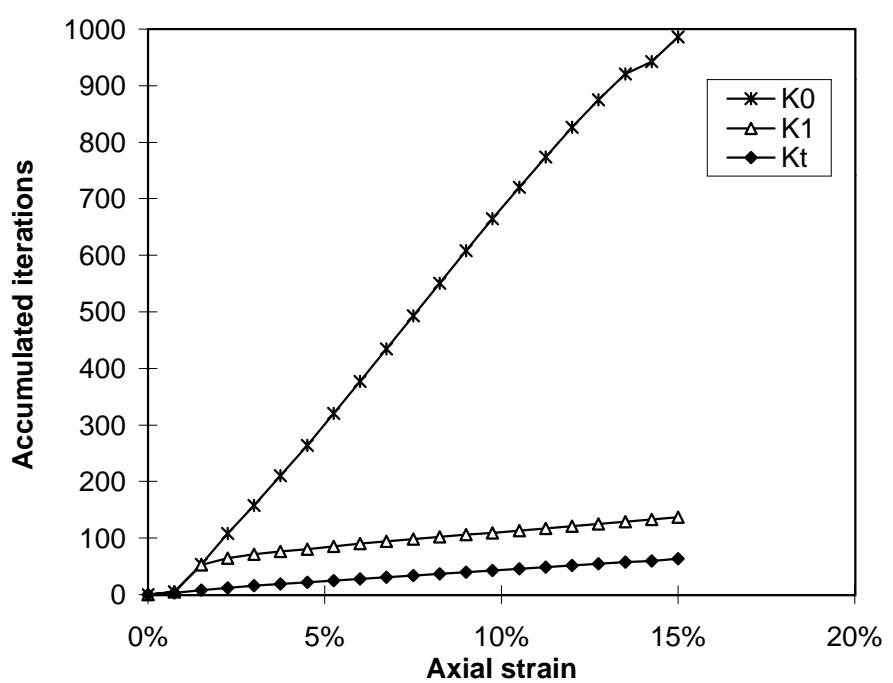

Figure 14. Accumulated iterations versus load level for the homogeneous triaxial problem with three nonlinear solvers: initial stress method (K0), modified Newton-Raphson method (K1) and full Newton-Raphson method (Kt)

In the following, attention is focused in the test with a precompression of $600 \mathrm{kPa}$. In Figure 14 three nonlinear solvers are compared, by showing the accumulated iterations versus the axial strain relationship. The advantage of using the consistent tangent matrix is clear: the number of iterations needed with the full (Kt) or modified (K1) Newton-Raphson methods is much lower 
than with the initial-stress (K0) method, that only uses the elastic tangent matrix. All the steps are in the plastic regime. However, Figure 14 clearly shows that the step with a convergence more sensible to the computation of the consistent tangent matrix is step 2. It is the first step with a significant change in the internal variables (more specifically, in $\kappa_{\text {con }}$ ). For that reason, the three differentiation schemes have been compared in that load step. The convergence results are shown in Figure 15, and the range of relative stepsizes that keep quadratic convergence is summarized in Table 9.
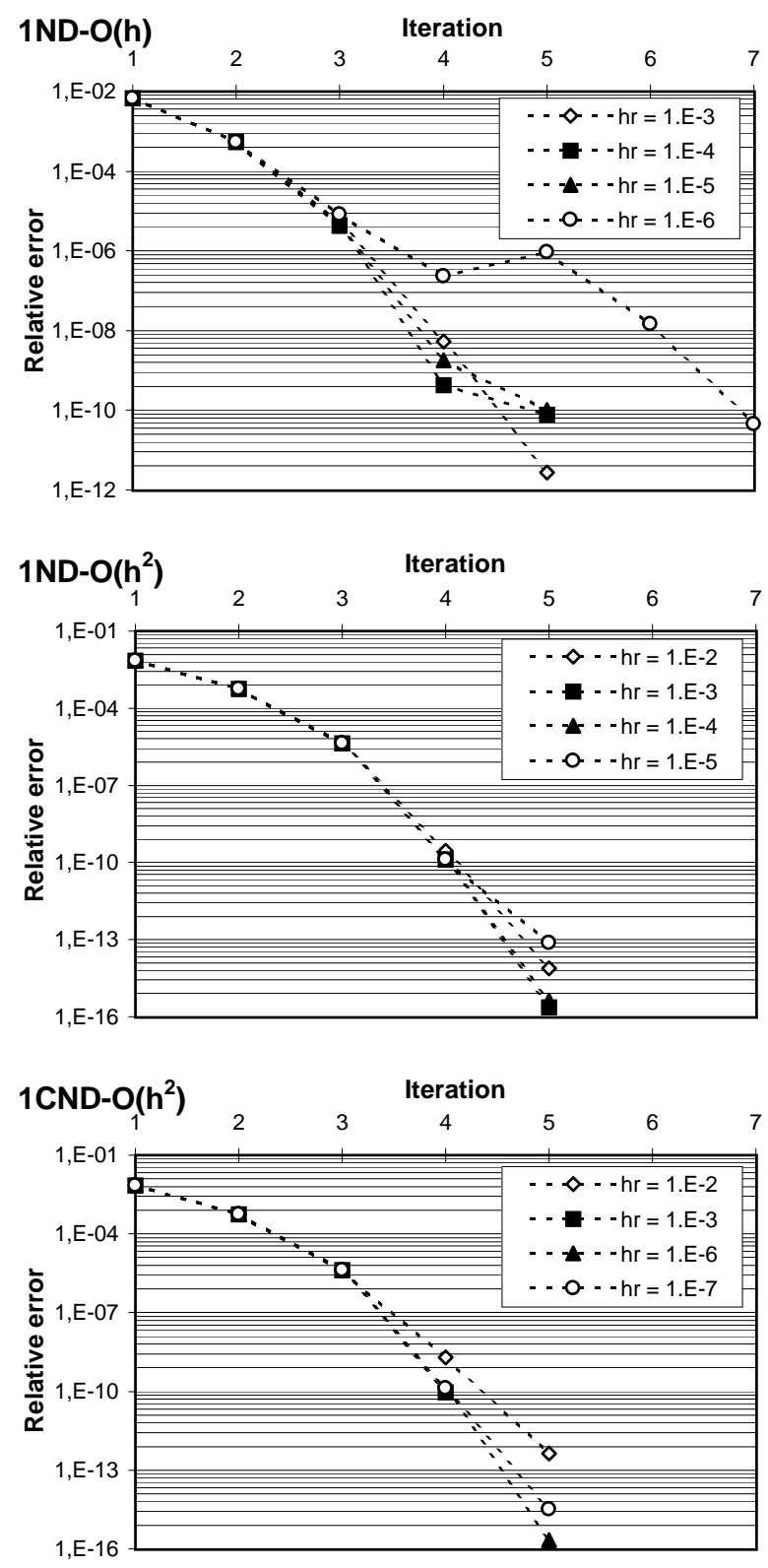

Figure 15. Convergence results for step 2 of the homogeneous triaxial problem with various relative stepsizes $h_{r}$ 
Table 9

\begin{tabular}{|l|l|}
\hline Num. approx. & Range of $h_{r}$ \\
\hline 1 ND-O $(h)$ & $10^{-3}-10^{-5}$ \\
\hline $1 \mathrm{ND}-\mathrm{O}\left(h^{2}\right)$ & $10^{-3}-10^{-5}$ \\
\hline $1 \mathrm{CND}-\mathrm{O}\left(h^{2}\right)$ & $10^{-3}-10^{-7}$ \\
\hline
\end{tabular}

Range of relative stepsizes $h_{r}$ that give quadratic convergence in the homogeneous triaxial problem up to a tolerance of $10^{-8}\left(1 \mathrm{ND}-\mathrm{O}(h)\right.$ approximation) or $10^{-10}$ (the other two)

The convergence results of Figure 15 show that the first-order scheme, 1ND$\mathrm{O}(h)$, keeps quadratic convergence up to a tolerance of $10^{-8}$, and that secondorder schemes reach a tolerance of $10^{-10}$. This difference is due to the fact that the Lode angle $\theta$ is equal to $\pi / 3$ for all the Gauss points (recall that the global problem is homogeneous). This region of stress space is the most demanding one for approximating the derivatives of the flow vector. For this reason, the differences between order 1 and order 2 in the truncation error are clear. However, it must be noted that the indicated tolerances are very strict for any practical application. Thus, even the 1ND-O $(h)$ approximation is accurate enough if a tolerance of, say, $10^{-6}$ is used. With this tolerance, the three techniques provide quadratic convergence for a wide range of relative stepsizes.

\subsubsection{Triaxial test: non-homogeneous sample}

The third example is the triaxial test with an non-homogeneous sample. A structured mesh of $600(20 \times 30)$ elements, the material parameters of soil S2 and a precompression of $600 \mathrm{kPa}$ have been used. The finite element in the the bottom left corner is weakened (the values of $\eta_{\text {con }}^{-}$and $p_{\text {cap }, 0}$ are $10 \%$ lower) to induce a non-homogeneous response.

The evolution of the second invariant of the deviatoric part of the strain tensor is depicted in Figure 16. Note that the axisymmetric nature of the test prevents localization [15].

Figure 17 shows the evolution of the load and the number of Gauss points which undergo plastic loading versus displacement. Note that after the limit load, a large reduction of number of Gauss points under plastic loading is found. Several Gauss points that were on plastic loading change to elastic unloading. Therefore, this part of the example is called partial unloading. During partial unloading, the mechanical behaviour of the sample becomes clearly non-homogeneous. 


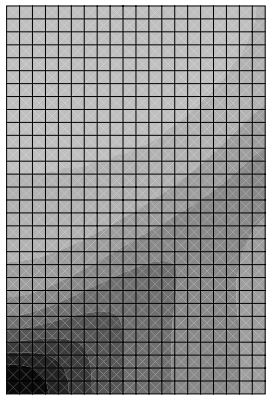

$3.5 \%$

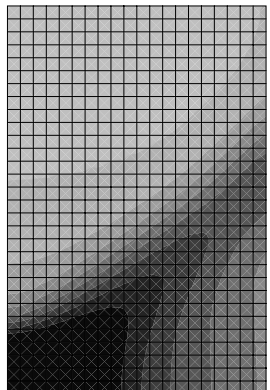

$4.0 \%$

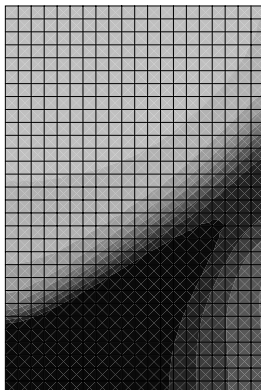

$4.5 \%$

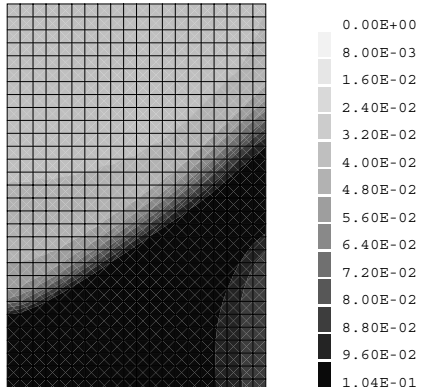

$5.0 \%$

Figure 16. Second invariant of the deviatoric part of $\varepsilon$ for different values of the axial strain (vertical displacement/initial height) in the non-homogeneous triaxial problem
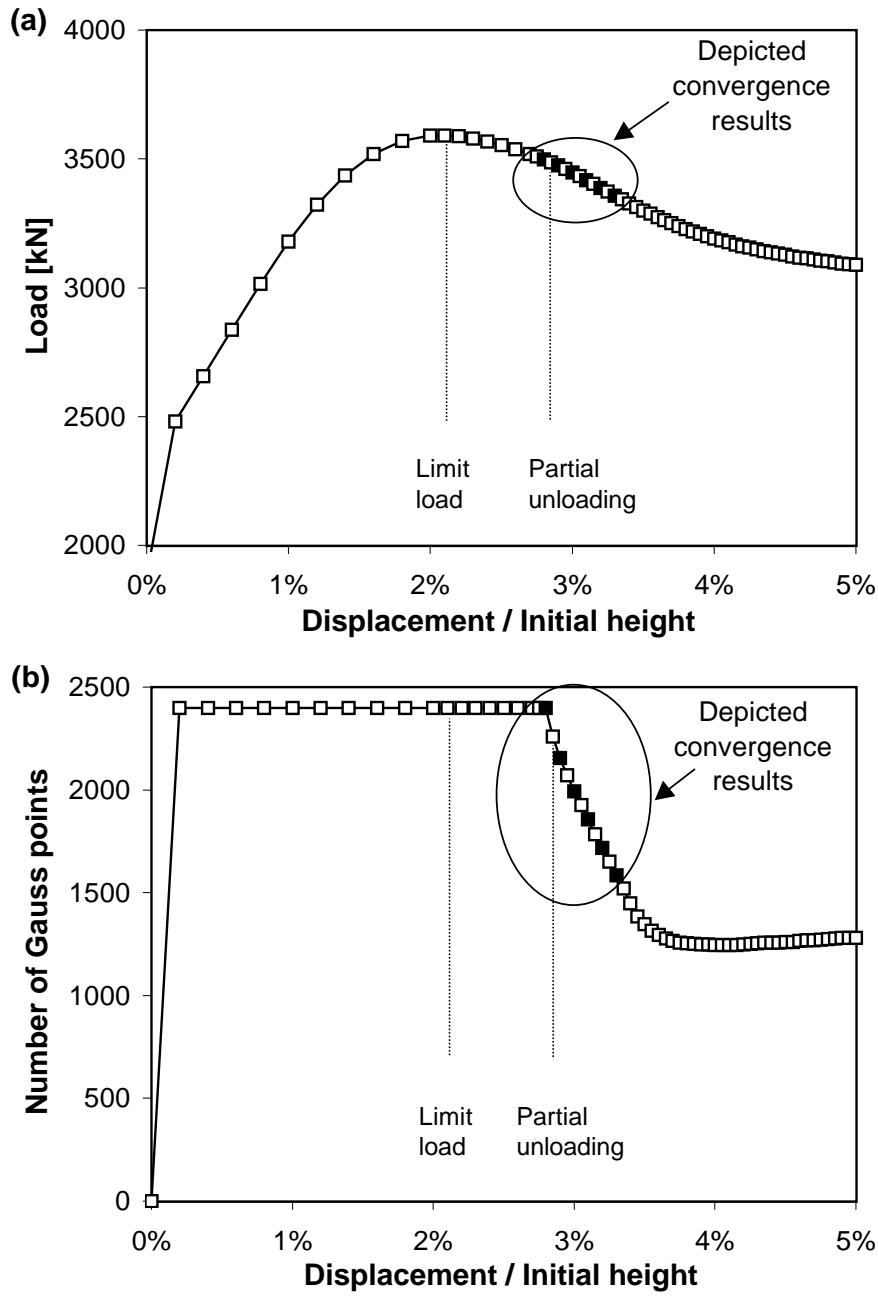

Figure 17. Non-homogeneous triaxial problem. Evolution of (a) load and (b) number of Gauss points with plastic loading versus displacement 


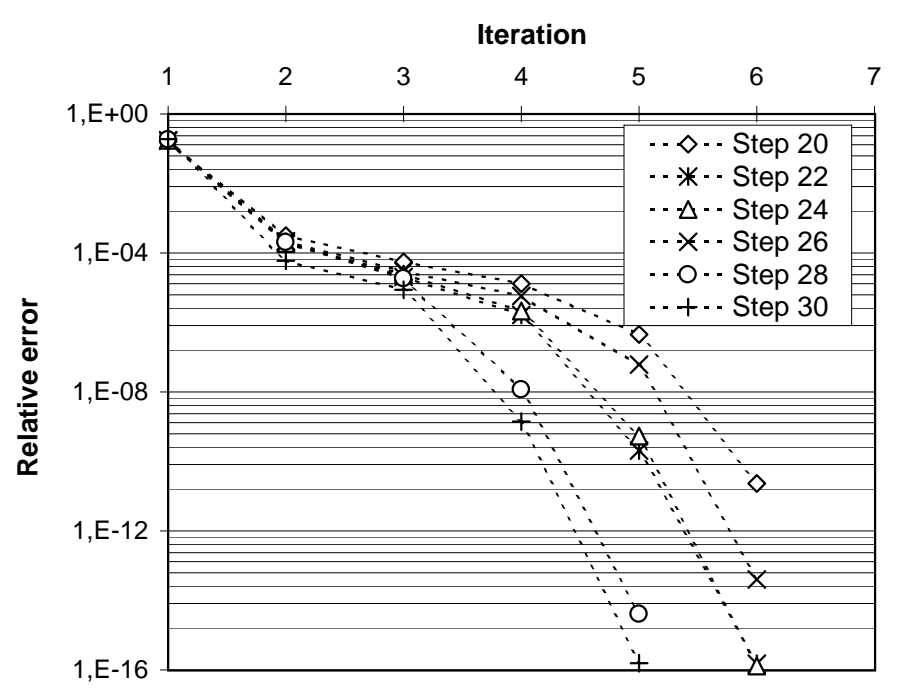

Figure 18. Convergence results for various load steps of the non-homogeneous triaxial problem

The convergence results for several load steps during partial unloading is depicted in Figure 18. Note that quadratic convergence is found. These results are obtained with the technique $1 \mathrm{CND}-\mathrm{O}\left(h^{2}\right)$, with $h_{r}=10^{-5}$ and with a tolerance of $10^{-10}$. In these steps, the same results are found with the other techniques and other relative stepsizes $h_{r}$.

However, if a too strict tolerance is imposed, the convergence results obtained during the hardening regime depend on the differentiation technique and the relative stepsize. It has been already shown for the homogeneous triaxial test that the first-order technique only ensures quadratic convergence up to a tolerance of $10^{-8}$. In order to check the behaviour of the three techniques with this example, the number of accumulated iterations along all the loading process with several $h_{r}$ are summarized in Table 10. Two tolerances are considered: $10^{-8}$ and $10^{-10}$.

Table 10(a) shows that, with a tolerance of $10^{-8}$, the three techniques need a very similar number of iterations for a wide range of relative stepsizes. This indicates quadratic convergence in all the entries of the table. On the other hand, only the 1CND-O $\left(h^{2}\right)$ approximation ensures quadratic convergence up to a tolerance of $10^{-10}$ during all the loading, see Table 10(b). For the other two approximations, there are variations in the number of iterations, caused by a loss of quadratic convergence.

A comparison of the computational cost (CPU time) of the three techniques with a tolerance of $10^{-10}$ and with several $h_{r}$ is presented in Table 11 . The values are given in $\%$ with respect to $1 \mathrm{ND}-\mathrm{O}(h)$ with $h_{r}=10^{-6}$. The forward difference scheme has a relative cost of $100-110 \%$, the centered difference 
scheme of $120-130 \%$ and the approximation based on complex variables of $130-135 \%$.

Finally, in Figure 19 several nonlinear solvers are compared. With the K0 method (which does not use the consistent tangent matrix) the step right after the limit load needs more than 400 iterations. The K1 method (consistent tangent matrix updated at the beginning of each step) fails to converge when the partial unloading is starting. Only with a full Newton-Raphson method, $\mathrm{Kt}$, or at least updating the consistent tangent matrix regularly within the step (each two iterations, Kd2, or each four, Kd4) can the analysis be completed.

(a)

(b)

\begin{tabular}{|c|c|c|c|c|c|c|}
\hline$h_{r}$ & $10^{-3}$ & $10^{-4}$ & $10^{-5}$ & $10^{-6}$ & $10^{-7}$ & $10^{-8}$ \\
\hline $1 \mathrm{ND}-\mathrm{O}(h)$ & 255 & 225 & 226 & 226 & 230 & - \\
$1 \mathrm{ND}-\mathrm{O}\left(h^{2}\right)$ & 225 & 227 & 226 & 225 & 228 & - \\
$1 \mathrm{CND}-\mathrm{O}\left(h^{2}\right)$ & 225 & 225 & 225 & 225 & 225 & 225 \\
\hline
\end{tabular}

Table 10

\begin{tabular}{|c|c|c|c|c|c|c|}
\hline$h_{r}$ & $10^{-3}$ & $10^{-4}$ & $10^{-5}$ & $10^{-6}$ & $10^{-7}$ & $10^{-8}$ \\
\hline $1 \mathrm{ND}-\mathrm{O}(h)$ & 315 & 271 & 268 & 251 & 260 & - \\
$1 \mathrm{ND}-\mathrm{O}\left(h^{2}\right)$ & 264 & 245 & 243 & 249 & 257 & - \\
$1 \mathrm{CND}-\mathrm{O}\left(h^{2}\right)$ & 253 & 236 & 235 & 235 & 239 & 236 \\
\hline
\end{tabular}

Number of accumulated iterations for the non-homogeneous triaxial problem: (a) with a tolerance of $10^{-8}$; (b) with a tolerance of $10^{-10}$

Table 11

\begin{tabular}{|c|c|c|c|c|c|c|}
\hline$h_{r}$ & $10^{-3}$ & $10^{-4}$ & $10^{-5}$ & $10^{-6}$ & $10^{-7}$ & $10^{-8}$ \\
\hline $1 \mathrm{ND}-\mathrm{O}(h)$ & $145 \%$ & $111 \%$ & $112 \%$ & $100 \%$ & $105 \%$ & - \\
$1 \mathrm{ND}-\mathrm{O}\left(h^{2}\right)$ & $150 \%$ & $122 \%$ & $119 \%$ & $122 \%$ & $127 \%$ & - \\
$1 \mathrm{CND}-\mathrm{O}\left(h^{2}\right)$ & $150 \%$ & $134 \%$ & $132 \%$ & $131 \%$ & $134 \%$ & $133 \%$ \\
\hline
\end{tabular}

Relative CPU time of the three numerical differentiation techniques with several relative stepsizes $h_{r}$ in the non-homogeneous triaxial problem up to a tolerance of $10^{-10}$

\section{Concluding remarks}

Numerical differentiation of the flow vector and the hardening moduli allows to compute the consistent tangent matrix when the analytical derivatives are not available. This allows to solve boundary value problems (i.e. global problems) in computational plasticity with quadratic convergence. 

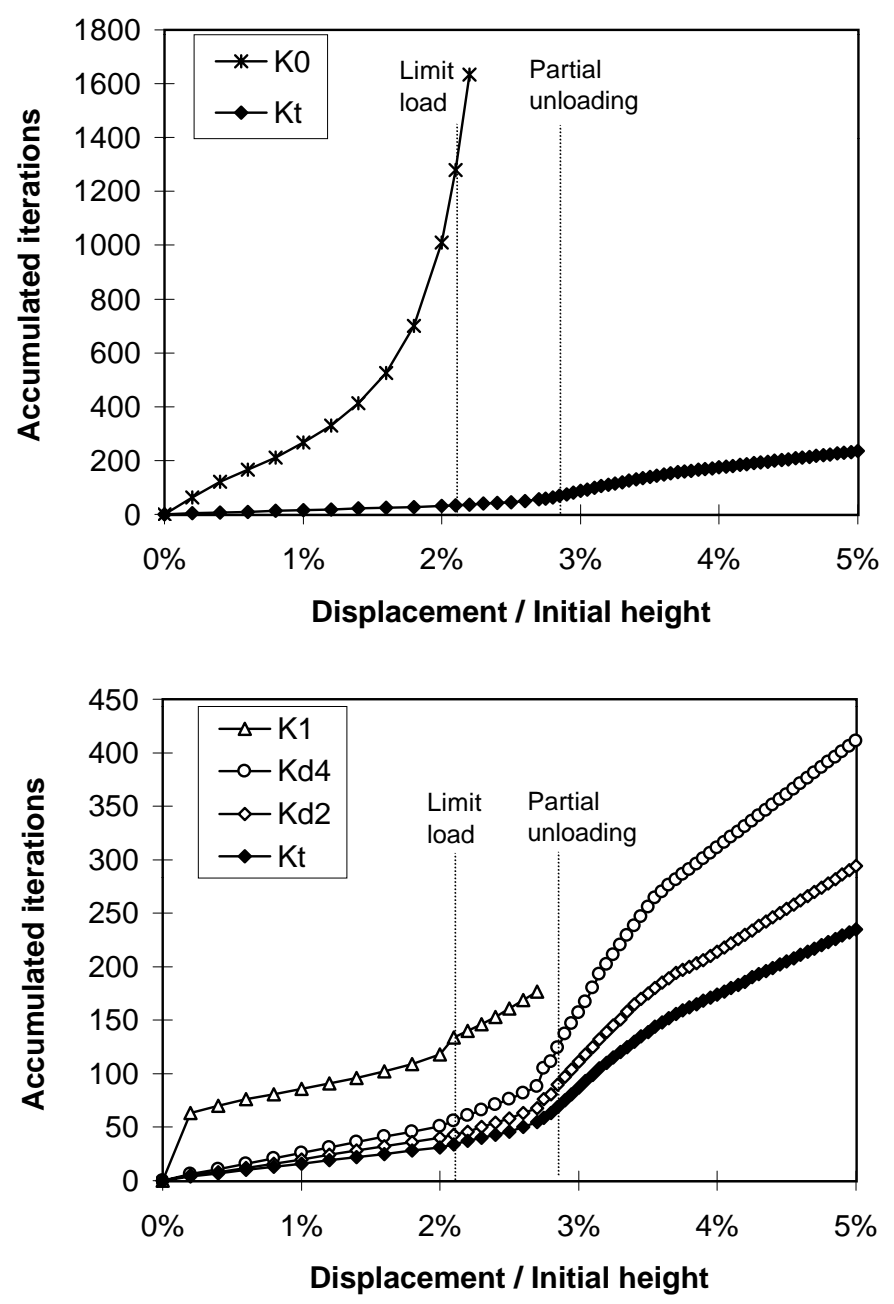

Figure 19. Accumulated iterations versus load level for the non-homogeneous triaxial problem with various nonlinear solvers

Moreover, the approximated derivatives are also used to compute the Jacobian of the residual of the local problem (time-integration of the elastoplastic constitutive equations at each Gauss point). Thus, a full Newton-Raphson method can be applied over stresses and internal variables, and quadratic convergence is also obtained.

The proposed approach has been illustrated with a complex material model: the MRS-Lade model. It is a cap-cone model with highly nonlinear hardening/softening laws. Analytical derivatives of the flow vector and the hardening moduli are not available in the literature. In fact, it is first time that quadratic convergence results are presented for this model (both for local and global problems). Extension to other complex material models is straightforward, and consists only in changing the definition of the flow vector and the hardening moduli. 
Three numerical differentiation schemes have been applied: forward and centered difference schemes (first and second order respectively) and an approximation based in complex variables (second order). The three schemes provide quadratic convergence. The choice of an adequate stepsize does not present any difficulty. The concept of relative stepsize introduced in Reference [1] has been exploited, and good results (i.e. quadratic convergence at the local and global level for a convergence tolerance of $10^{-8}$ or even more strict) have been obtained for a wide range of relative stepsizes.

As a final concluding remark, it must be noted that the proposed approach has a modest computational cost. A direct comparison of analytical versus numerical differentiation cannot be made for this model, because analytical derivatives are not available. However, for other material models, an overhead in computational cost (of numerical derivatives with respect to analytical derivatives) of only $1 \%$ to $2 \%$ has been found, see [1].

\section{References}

[1] A. Pérez-Foguet, A. Rodríguez-Ferran, and A. Huerta. Numerical differentiation for local and global tangent operators in computational plasticity. Accepted for publication in Computer Methods in Applied Mechanics and Engineering, 1998.

[2] S. Sture, K. Runesson, and E. J. Macari-Pasqualino. Analysis and calibration of a three-invariant plasticity model for granular materials. Ingenieur Arquiv, 59:253-266, 1989.

[3] B. Jeremić and S. Sture. Implicit integrations rules in plasticity: Theory and implementation. Technical report, Report to NASA Marshall Space Flight Center, Contract: NAS8-38779, University of Colorado at Boulder, Jul. 1994.

[4] J. C. Simo and R. L. Taylor. Consistent tangent operators for rate-independent elastoplasticity. Computer Methods in Applied Mechanics and Engineering, 48:101-118, 1985.

[5] E. J. Macari, K. Runesson, and S. Sture. Response prediction of granular materials at low effective stresses. Journal of Geotechnical Engineering, 120:1252-1268, 1994.

[6] B. Jeremić and S. Sture. Implicit integrations in elastoplastic geotechnics. Mechanics of Cohesive-Frictional Materials, 2:165-183, 1997.

[7] E. J. Macari, S. Weihe, and P. Arduino. Implicit integration of elastoplastic constitutive models for frictional materials with highly non-linear hardening functions. Mechanics of Cohesive-Frictional Materials, 2:1-29, 1997.

[8] M. Ortiz and E. P. Popov. Accuracy and stability of integration algorithms for elastoplastic constitutive relations. International Journal for Numerical Methods in Engineering, 21:1561-1576, 1985. 
[9] J. C. Simo and T. J. R. Hughes. Computational inelasticity. Springer-Verlag, 1998.

[10] A. Pérez-Foguet and A. Huerta. Plastic flow potential for the cone region of the MRS-Lade model. Journal of Engineering Mechanics, 125(3):364-367, 1999.

[11] M. A. Crisfield. Non-linear finite element analysis of solids and structures. 1 Essentials. John Wiley \& Sons, Chichester, 1991.

[12] K. Runesson, A. Samuelsson, and L. Bernspang. Numerical technique in plasticity including solution advancement control. International Journal for Numerical Methods in Engineering, 22:769-788, 1986.

[13] M. Ortiz and J. B. Martin. Symmetry-preserving return mapping algorithms and incrementally extremal paths: a unification of concepts. International Journal for Numerical Methods in Engineering, 28:1839-1853, 1989.

[14] D. M. Potts and A. Gens. A critical assessment of methods of correcting for drift from the yield surface in elasto-plastic finite element analysis. International Journal for Numerical and Analytical Methods in Geomechanics, 9:149-159, 1985 .

[15] J.W. Rudnicki and J.R. Rice. Conditions for the localization of deformation in pressure-sensitive dilatant materials. Journal of the Mechanics and Physics of Solids, 23:371-394, 1975.

\section{A Appendix}

In the following, the formulas needed to compute the flow vector and the hardening/softening moduli that have not been presented in Subsection 2.2 are summarized.

The model is expressed through the following three invariants:

$$
p=-\frac{1}{3} I_{1} \quad q=\sqrt{3 J_{2}} \quad \theta=\frac{1}{3} \arccos \left(\frac{3 \sqrt{3} J_{3}}{2 J_{2}^{3 / 2}}\right)
$$

with

$$
I_{1}=\boldsymbol{\sigma}^{t} \boldsymbol{\delta} \quad J_{2}=\frac{1}{2} \mathbf{s}^{t} \mathbf{L} \mathbf{s} \quad J_{3}=\operatorname{det}[\mathbf{s}]
$$

where $\mathbf{s}=\boldsymbol{\sigma}+p \boldsymbol{\delta}, \mathbf{L}$ is a diagonal matrix with the diagonal terms equal to $\{1,1,1,2,2,2\}$ (in three-dimensional problems), and $\operatorname{det}[*]$ is the determinant of $*$.

The expression of the Willam-Warnke function reads

$$
g(\theta)=\frac{(2 e-1)^{2}+4\left(1-e^{2}\right) \cos (\theta)^{2}}{2\left(1-e^{2}\right) \cos (\theta)-(2 e-1) \sqrt{5 e^{2}-4 e+4\left(1-e^{2}\right) \cos (\theta)^{2}}},
$$


where $e$ is a parameter of the model.

The expressions of the hardening/softening laws are

$$
\begin{aligned}
& \eta_{\text {con }}\left(\kappa_{\text {con }}\right)=a \exp \left(-b \kappa_{\text {con }}\right)\left(k_{1}+\kappa_{\text {con }}\right)^{1 / v}+k_{2} \eta_{\text {con }}^{-}\left(\frac{\kappa_{\text {con }}}{\epsilon+\kappa_{\text {con }}}\right) \\
& p_{\text {cap }}\left(\kappa_{\text {cap }}\right)=p_{\text {cap }, 0}\left(1+\kappa_{\text {cap }}^{1 / r}\right)
\end{aligned}
$$

where $k_{1}, k_{2}, v, \eta_{\text {con }}^{-}$and $\epsilon$ are parameters of the model, and

$$
\begin{aligned}
a & =\exp (b)\left(\frac{1}{1+k_{1}}\right)^{1 / v}\left(1-\frac{k_{2}}{1+\epsilon}\right) \eta_{\mathrm{con}}^{-} \\
b & =\frac{1}{v\left(1+k_{1}\right)}+\frac{k_{2} \epsilon}{(1+\epsilon)\left(1+\epsilon-k_{2}\right)} .
\end{aligned}
$$

Finally, $\mathbf{n}_{\text {con }}$ and $\mathbf{n}_{\text {cap }}$, the derivatives of $F_{\text {con }}$ and $F_{\text {cap }}$ with respect to $\sigma$, are obtained applying the chain rule:

$$
\frac{\partial F_{*}}{\partial \boldsymbol{\sigma}}=\frac{\partial F_{*}}{\partial p} \frac{\partial p}{\partial \boldsymbol{\sigma}}+\frac{\partial F_{*}}{\partial q} \frac{\partial q}{\partial \boldsymbol{\sigma}}+\frac{\partial F_{*}}{\partial \theta} \frac{\partial \theta}{\partial \boldsymbol{\sigma}}
$$

where the subscript ${ }^{*}$ stands for either cone or cap.

The derivatives of $p, q$ and $\theta$ with respect to $\sigma$ are

$$
\begin{aligned}
& \frac{\partial p}{\partial \boldsymbol{\sigma}}=-\frac{1}{3} \boldsymbol{\delta} \\
& \frac{\partial q}{\partial \boldsymbol{\sigma}}=\frac{3}{2 q} \mathbf{L} \mathbf{s} \\
& \frac{\partial \theta}{\partial \boldsymbol{\sigma}}=\left(\frac{3 \cos (3 \theta)}{2 q^{2} \sin (3 \theta)}\right) \mathbf{L} \mathbf{s}+\left(\frac{9}{2 q^{3} \sin (3 \theta)}\right) \frac{\partial J_{3}}{\partial \boldsymbol{\sigma}} .
\end{aligned}
$$

The partial derivatives of $F_{\text {con }}$ are

$$
\begin{aligned}
& \frac{\partial F_{\text {con }}}{\partial p}=-\eta_{\text {con }}\left(\kappa_{\text {con }}\right) \\
& \frac{\partial F_{\text {con }}}{\partial q}=\left(1+\frac{q}{q_{\mathrm{a}}}\right)^{m}\left(1+\frac{m q}{q_{\mathrm{a}}+q}\right) g(\theta) \\
& \frac{\partial F_{\text {con }}}{\partial \theta}=q\left(1+\frac{q}{q_{\mathrm{a}}}\right)^{m} \frac{\partial g(\theta)}{\partial \theta}
\end{aligned}
$$


and the partial derivatives of $F_{\text {cap }}$ are

$$
\begin{aligned}
& \frac{\partial F_{\text {cap }}}{\partial p}=2 \frac{p-p_{\mathrm{m}}}{p_{\mathrm{r}}^{2}} \\
& \frac{\partial F_{\text {cap }}}{\partial q}=\frac{2 q g(\theta)^{2}}{f_{\mathrm{r}}^{2}}\left(1+\frac{q}{q_{\mathrm{a}}}\right)^{2 m}\left(1+\frac{m q}{q_{\mathrm{a}}+q}\right) \\
& \frac{\partial F_{\text {cap }}}{\partial \theta}=\frac{2 q^{2} g(\theta)}{f_{\mathrm{r}}^{2}}\left(1+\frac{q}{q_{\mathrm{a}}}\right)^{2 m} \frac{\partial g(\theta)}{\partial \theta} .
\end{aligned}
$$

